\title{
FLORA Y VEGETACIÓN DE LAS DUNAS DE LA PLAYA GRANDE DE CARTAGENA, UNA PLAYA URBANA EN CHILE CENTRAL
}

\author{
Andrés Madrid' ${ }^{1}$ iD \& Sebastián Teillier ${ }^{2}$
}

${ }^{1}$ Consultor independiente. Los Militares 5953, Of. 801, Las Condes, Región Metropolitana, Chile; andresmadridp@gmail.com (autor corresponsal).

${ }^{2}$ Escuela de Arquitectura y Paisaje, Universidad Central de Chile. Santa Isabel 1186, Santiago, Región Metropolitana, Chile.

\begin{abstract}
Madrid, A. \& S. Teillier. 2021. Flora and vegetation of the dunes of Playa Grande of Cartagena, an urban beach in central Chile. Darwiniana, nueva serie 9(2): 342-363.

The flora of the Playa Grande of Cartagena in the Valparaiso Region, commune of El Tabo ( $33^{\circ} 30^{\prime} \mathrm{S}$, $71^{\circ} 36^{\prime} \mathrm{W}$ ), is made up of 98 species of vascular plants, $61 \%$ of which are exotics, most feral. The vegetation is made up of five units: a Carpobrotus chilensis and Chrysanthemum coronarium grassland dominated by ruderal species, mostly feral alien species, which marks the boundary between the populated area and the dune field with the sandy beach; a Ambrosia chamissonis and Poa cumingii scrub that is typical of the primary and secondary dunes and occupies the largest vegetated area in the study area; a Nolana crassulifolia scrub, a coastal rocky rocks; and two units typical of coastal wetlands: a marsh grassland with Schoenoplectus pungens and Cotula coronopifolia, and a Typha dominguensis grassland in a water body.
\end{abstract}

Keywords. Dunes; flora of Chile, urban coastal wetlands.

Resumen. Madrid, A. \& S. Teillier. 2021. Flora y vegetación de las dunas de la Playa Grande de Cartagena, una playa urbana en Chile central. Darwiniana, nueva serie 9(2): 342-363.

La flora de la Playa Grande de Cartagena, en la Región de Valparaíso, comuna de El Tabo (3330’'S, $71^{\circ} 36^{\prime} \mathrm{W}$ ), reúne a 98 especies de plantas vasculares, de las que un $61 \%$ son exóticas, la mayoría asilvestradas. La vegetación está constituida por cinco unidades: un herbazal de Carpobrotus chilensis y Chrysanthemum coronarium dominado por especies ruderales, en su mayoría alóctonas asilvestradas, que señala el límite entre la zona poblada y el campo de dunas con la playa de arena; un matorral de Ambrosia chamissonis y Poa cumingii que es propio de las dunas primarias y secundarias y ocupa la mayor superficie vegetada de la zona de estudio; un matorral de Nolana crassulifolia, comunidad rupícola litoral; y dos unidades propias de los humedales del litoral: un herbazal de marisma con Schoenoplectus pungens y Cotula coronopifolia, y un herbazal de Typha dominguensis en un espejo de agua.

Palabras clave. Dunas; flora de Chile; humedales costeros urbanos.

\section{INTRODUCCIÓN}

La flora y la vegetación de Chile central presentan niveles importantes de invasión o de pérdida de superficie. Al menos un $32 \%$ de las 934 especies de la flora vascular del litoral de la Región de Valparaíso son alóctonas asilvestradas (Teillier et al., 2018). Al nivel de la vegetación, solo se conocen datos generales sobre el estado actual de los ecosistemas de la región; al respecto, se reconoce la pérdida de hasta un $25 \%$ de la superficie de la vegetación nativa litoral caracterizada como un matorral esclerófilo arborescente de Peumus boldus Molina (Monimiaceae) y Schinus latifolius (Gillies ex Lindl.) Engler (Anacardiaceae) (Luebert \& Pliscoff, 2017). 
Estudios en Europa, Norteamérica, China y Chile han mostrado que las ciudades o las áreas urbanas y su periferia son lugares con una importante riqueza de plantas exóticas asilvestradas (Pyšek, 1998; Clemants \& Moore, 2003; Zhao et al., 2010; Figueroa et al., 2016). En particular, esto también se observó en las localidades ubicadas cerca de las playas y de las dunas litorales, que son ambientes susceptibles de ser invadidos por las plantas alóctonas tal como se ha descrito en México, Australia, España y Sudáfrica (Castillo \& Moreno, 1996; Heyligers, 2008; Asensi et al., 2014; Cowling et al., 2019). Relacionado con ello, Jehlík et al. (2019) mostraron que los recintos de los puertos fluviales de Europa central contienen una alta proporción de especies exóticas asilvestradas (41\%), cuya abundancia aumenta con la superficie del puerto, siendo éstos importantes núcleos de dispersión de especies alóctonas asilvestradas hacia el interior de los países.

Las dunas litorales son ecosistemas que se ubican en la transición entre los ambientes terrestres y marinos y son parte, a su vez, del sistema de reparto de arena formado por la playa, de gran movilidad, y las dunas más estables (Martínez \& Psuty, 2004). Como resultado de la interacción entre las características geomorfológicas, la heterogeneidad ambiental y la diversidad de las especies, las dunas costeras albergan una importante diversidad ecológica, proporcionando bienes y servicios importantes que se han utilizado durante mucho tiempo para numerosas actividades humanas, siendo sustancialmente alteradas por la actividad humana y muchas de ellas ya están severa e irreversiblemente degradadas (Martínez \& Psuty, 2004). Hay que considerar que en los sistemas de dunas litorales, cuando están bien conservados, las plantas atrapan la arena y les brindan estabilidad a las dunas (Calvã et al., 2017). En Chile central, las dunas litorales se encuentran en todo el largo de la costa del océano Pacifico y su origen está relacionado con modificaciones climáticas y del nivel del mar durante el Cuaternario, atribuyéndoseles un carácter formativo permanente (Paskoff, 1970). En los ecosistemas con clima de tipo mediterráneo, las dunas costeras del Holoceno se caracterizan por ser pequeñas, fragmentadas y dinámicas, con suelos arenosos, secos y desequilibrados nutricionalmente (Cowling et al., 2019). Estas características, junto con la sequía estival y los vientos cargados de sal, plantean muchos desafíos para la colonización y la persistencia de las plantas. Se sugiere que las dunas de Sudáfrica son las menos similares a las de la cuenca del Mediterráneo, donde las herbáceas anuales son la forma de crecimiento más frecuente, mientras que los arbustos están subordinados. Por su parte, las floras de las dunas de California y Chile central parecen ocupar una posición intermedia, en términos de mezcla de formas de crecimiento, entre El Cabo y Australia, donde las floras de dunas han conservado características típicas de suelos pobres en nutrientes, y la cuenca del Mediterráneo, donde los arbustos enanos y caducifolios, junto con las hierbas anuales dominan el espectro de formas de vida (Cowling et al., 2019).

Todas las dunas de los ecosistemas con clima del tipo mediterráneo están amenazadas por la invasión de plantas exóticas, el desarrollo de infraestructura, la demanda turística y el aumento del nivel del mar (Cowling et al., 2019).

La flora de las dunas litorales de Chile central reuniría, entre nativas y alóctonas, a unas 186 especies de plantas vasculares (San Martín et al., 1992a), con un $30 \%$ de especies alóctonas asilvestradas. Sin embargo, en estos ecosistemas dunarios también crecen algunas especies endémicas del litoral central de Chile, de las que al menos dos se conoce que están amenazadas, Astragalus trifoliatus y Oenothera grisea, las que fueron clasificadas oficialmente como "en peligro crítico" (MMA, 2018). La primera de ellas se considera endémica del campo de dunas "Gota de Leche" (Teillier \& Zuloaga, 2016), ubicado en la comuna de El Tabo, provincia de San Antonio, al norte del balneario de Las Cruces; en tanto que la segunda, es endémica de las dunas de Concón y de Quintero (Dietrich, 1977), ambas ubicadas en la Región de Valparaíso.

Las 186 especies de plantas vasculares descritas para las dunas litorales de Chile 
central ocupan todas las formas de vida del espectro de Raunkiaer, dominando entre ellas las terófitas y las hemicriptófitas con un $38 \%$ y $24 \%$, respectivamente (San Martín et al. 1992a). Flores \& Araya (2015) reportaron para las dunas de Ritoque, Región de Valparaíso, unas 121 especies, de las que un $25 \%$ eran hemicriptófitas y un $20 \%$ terófitas, mientras que para el humedal de Putú, en la Región del Maule, Urrutia (2016) documentó 110 taxones con un $39 \%$ de hemicriptófitas y un $29 \%$ de terófitas.

El marco de referencia para el estudio de la vegetación de las dunas de América del Sur en general, de Chile en general y de Chile central en particular, fue establecido por Kohler (1970, 1971), Eskuche (1992) y Ramírez et al. (1992). Por su parte, la vegetación de los humedales costeros fue estudiada por Ramírez \& Álvarez (2012) y de las marismas del centrosur de Chile fue revisada por San Martín et al. (1992b). Entre los estudios locales sobre la flora y vegetación de las dunas de Chile se mencionan Donoso (1974) en las dunas de Llico, Caldichoury (2000) y Flores \& Arancibia (2017) en las dunas de Santo Domingo-El Yali y Luebert \& Muñoz (2005) en las dunas de Concón. Por otra parte, la flora y la vegetación de las desembocaduras de los esteros y ríos de Chile central, los que con frecuencia incluyen parches de dunas, fue estudiada en el estero Reñaca-Viña del Mar (San Martín et al., 2001), estero Casablanca (Iturriaga, 2004), quebrada de Córdova-El Tabo (Pérez \&Villagrán, 1985), río Aconcagua (Arancibia, 2006), quebrada de La Hoyada (Brito, 2017) y laguna El Peral (Ramírez et al., 1987).

En el contexto descrito, la localidad de Cartagena, el balneario más antiguo de Chile central muestra un paisaje vegetal altamente intervenido y modificado, razón por la que se han iniciado estudios para establecer las prioridades locales de conservación a nivel de la flora y la vegetación. Al respecto, y dado que no existe una caracterización particular de las dunas litorales de dicha localidad, el objetivo de este trabajo es llevar a cabo una descripción de la flora vascular y de las comunidades de vegetación de sus dunas costeras.

\section{MATERIALES Y MÉTODOS}

\section{Área de estudio}

El área de estudio se localiza en un entorno urbano y corresponde al tramo norte de la playa Grande de Cartagena (Fig. 1). El área se extiende entre el balneario de Las Cruces y el estero La Cigüeña, en el sector de Costa Azul, comuna de El Tabo, provincia de San Antonio, Región de Valparaíso, Chile $\left(33^{\circ} 30^{\prime} \mathrm{S}, 71^{\circ} 36^{\prime} \mathrm{W}\right)$. El área analizada alcanza una longitud aproximada de $2,7 \mathrm{~km}$, con un ancho de 20 a $120 \mathrm{~m}$. En ella se distinguen tres tramos con contextos y bordes diferentes. El primero de norte a sur, es un sector con viviendas que pertenece al balneario de Las Cruces y se inserta en la parte sur de la zona típica Barrios Vaticano y Quirinal (D.S. $\mathrm{N}^{\circ} 110 / 2015$, del Mineduc), alcanza una longitud de $370 \mathrm{~m}$ y es el único tramo que tiene calzada y vereda pavimentadas; el segundo, corresponde al condominio de edificios Ilimay y tiene una longitud de $390 \mathrm{~m}$, no tiene acceso vehicular y tiene una calzada peatonal de tierra; y finalmente, el tercero, tiene una longitud de $1900 \mathrm{~m}$, no posee veredas, tiene una calzada vehicular de tierra y reúne a los sectores de Playas Blancas y San Carlos en la denominada Costa Azul, zona densamente poblada con viviendas bajas. El área de estudio comprendió 22 ha, de las que 8,51 ha correspondían a superficie vegetada naturalmente y el resto se encontraba sin vegetación.

El área de estudio se ubica en el denominado "Litoral Central" (Castro \& Hidalgo, 2002) que es como se conoce la zona costera entre las localidades de Algarrobo y de Santo Domingo. En 1954 el área mostraba un $20 \%$ de su superficie con uso urbano, que aumentó a $60 \%$ hacia el año 2000; en dicho periodo el $67 \%$ del aumento de la superficie urbanizada se materializó sobre las dunas (Castro \& Hidalgo, 2002), siendo por ello el medio natural que sufrió el mayor impacto por el crecimiento urbano. En relación con ello, para el periodo 1963-2017, se reportó que la degradación de los tres tipos de dunas en Cartagena había sido muy notoria: la paleoduna, había perdido un $94 \%$ de superficie; la de tipo "parabólica", un $54 \%$ y la denominada "anteduna de borde", un 27 \% (Ugalde, 2018). 
A. MADRID \& S. TEILLIER. Flora y vegetación de la Playa Grande de Cartagena, Chile

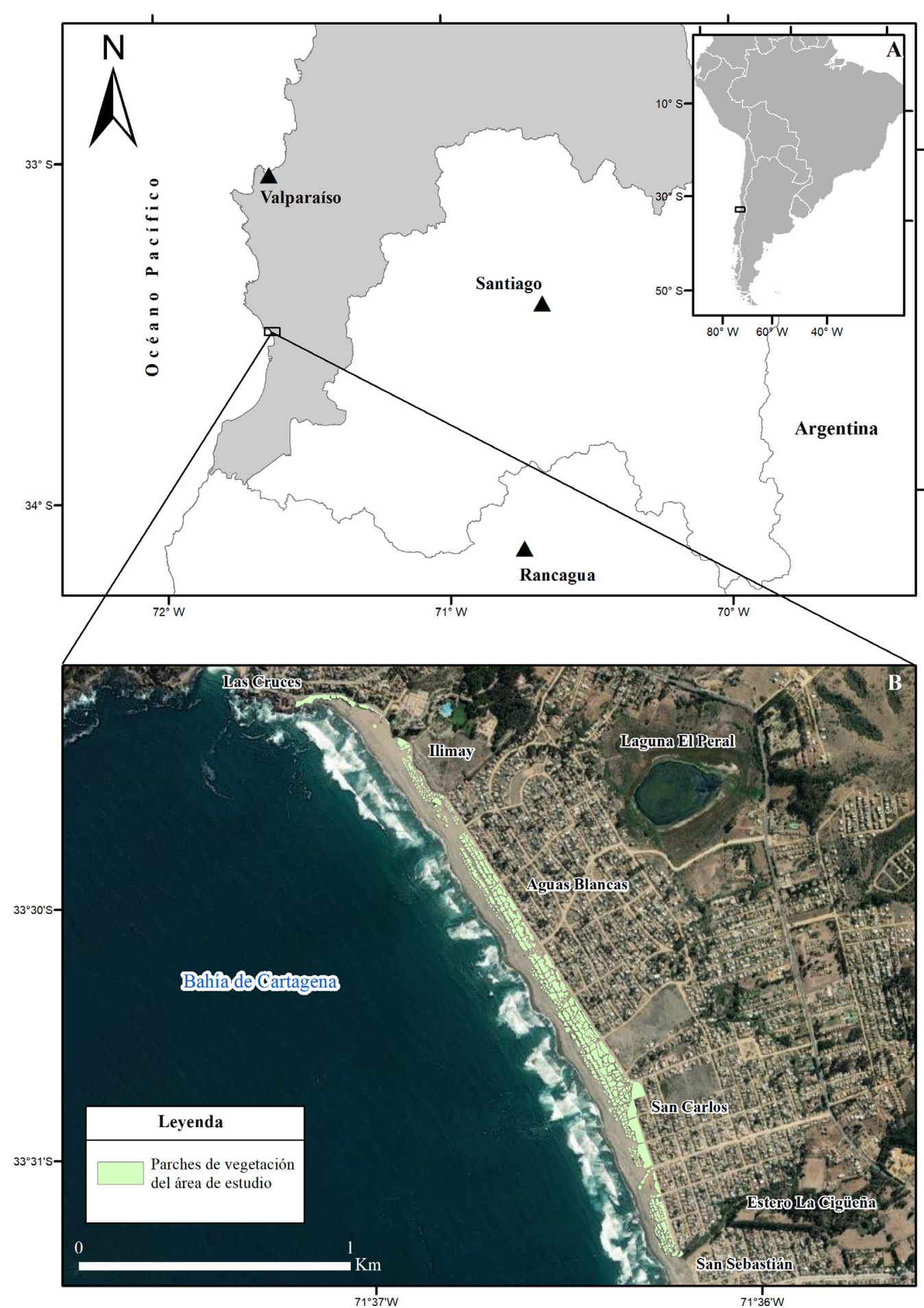

Fig. 1. Ubicación del área de estudio. A, Región de Valparaíso, Chile. B, Playa Grande de Cartagena, tramo norte, comuna de El Tabo, provincia de San Antonio. Fuente de la imagen satelital: Esri, DigitalGlobe, Geoeye, Earthstar Geographics, CNES/Airbus DS, USDA, USGS, AeroGRID, IGN, GIS User Community. Figura en color en la versión en línea http://www.ojs.darwin.edu.ar/index.php/darwiniana/article/view/959/1238 


\section{Métodos de flora}

El catálogo de la flora vascular se obtuvo mediante recorridos exhaustivos en el área de estudio, incorporando a él la información de 26 inventarios levantados en los diferentes tipos de vegetación. La información se obtuvo en cuatro campañas llevadas a cabo durante los meses de julio, octubre, noviembre y diciembre de 2016. La nomenclatura y origen geográfico de las especies sigue a Rodríguez et al. (2018). A cada especie se le asignó una forma de vida de Raunkiaer (1934) y la distribución geográfica original según Fuentes et al. (2020). Se recolectaron y herborizaron plantas de la mayor parte de las especies. Las plantas se determinaron en el campo o en gabinete utilizando la bibliografía taxonómica pertinente. El material recolectado en este trabajo está depositado en el herbario de CONC (siglas según Thiers, 2021). En el Apéndice 1 se detalla la lista completa de especies con sus autores.

\section{Métodos de vegetación}

Para clasificar y caracterizar las comunidades de vegetación se utilizó la metodología propuesta por la escuela fitosociológica de Zurich-Montpellier (o de Braun-Blanquet), la que se basa en levantar información en terreno mediante relevamientos fitosociológicos (censos) cuyo requisito fundamental es que cada uno se localice en sitios que posean condiciones de homogeneidad florística, fisonómica y ecológica (Müeller-Dombois \& Ellenberg, 1974). Para sistematizar el muestreo, el área de estudio se clasificó en gabinete mediante la interpretación a diferentes escalas de la imagen satelital de Google Earth Image ${ }^{T M}$ (C2016 Maxar Technologies, con base en la que se establecieron las unidades homogéneas sensu Braun Blanquet (1979). La superficie de los inventarios en dichas áreas homogéneas se determinó con base en el área mínima para cada tipo de vegetación y en su mayoría alcanzó a valores en torno a $100 \mathrm{~m}^{2}$. Las parcelas de inventario (censos) se dispusieron de modo que se cubriera toda el área de estudio, levantándose finalmente 26 parcelas (tres sin registros de flora), correspondientes a una superficie de 0,26 ha, en las que estaban representadas todos los tipos de homogeneidad.
Tabla 1. Índice de Abundancia - Dominancia (BraunBlanquet, 1979).

\begin{tabular}{|c|c|}
\hline Índice & $\begin{array}{r}\text { Porcentaje de cobertura-abundancia } \\
\hline 5\end{array}$ \\
\hline 4 & $\begin{array}{r}\text { Cualquier número de individuos, } \\
\text { cubre más del } 75 \% \text { de la superficie }\end{array}$ \\
\hline 3 & $\begin{array}{r}\text { Cualquier número de individuos, } \\
\text { cubre entre el } 50 \text { y } 75 \% \text { de la superficie }\end{array}$ \\
\hline 2 & $\begin{array}{r}\text { Cualquier número de individuos, } \\
\text { cubre entre el } 25 \text { y } 50 \% \text { de la superficie }\end{array}$ \\
\hline 1 & $\begin{array}{r}\text { Cualquier número de individuos, } \\
\text { cubre entre el } 5 \text { y } 25 \% \text { de la superficie }\end{array}$ \\
\hline+ & Abundante, cubre entre el 1 y $5 \%$ de la superficie \\
\hline $\mathrm{r}$ & Poco abundante, cubre $<1 \%$ \\
\hline
\end{tabular}

Los inventarios son posteriormente clasificados mediante metodologías particulares que ponen en evidencia a las especies consideradas como características o indicadoras de cada tipo de vegetación (Braun-Blanquet, 1979; Knapp, 1984). El relevamiento en campo consistió en anotar las especies y luego, asignarles una cobertura mediante una estimación visual directa utilizando la escala de frecuencia/abundancia según se muestra en la Tabla 1 (Braun-Blanquet, 1979); las especies que se observaron fuera de los límites de las parcelas, pero en la misma unidad, fueron indicadas como " $(+)$ ". Las 26 parcelas relevadas se clasificaron agrupando los censos según las especies dominantes y las características locales, definiéndolas como las especies más frecuentes en una comunidad, ausentes o con baja abundancia o frecuencia en las demás.

\section{RESULTADOS}

\section{Flora}

La riqueza de la flora alcanzó a 98 especies de plantas vasculares. La lista de ellas indicando la familia, el nombre científico, la forma de vida de Raunkiaer, el origen geográfico y su distribución original se muestra en el Apéndice 1. Las 98 especies se incluyen en 81 géneros y 29 familias. Las familias con mayor número de especies fueron Asteraceae, Poaceae, Brassicaceae y Fabaceae s.l. (Tabla 2), las que en conjunto alcanzaron a un $48 \%$ de la riqueza (Tabla 2), con entre cinco y 
Tabla 2. Familias con tres o más especies.

\begin{tabular}{|c|c|c|c|}
\hline Familia & $\begin{array}{c}\text { Especies } \\
\text { autóctonas }\end{array}$ & $\begin{array}{c}\text { Especies } \\
\text { alóctonas }\end{array}$ & Total \\
\hline Asteraceae & 3 & 15 & 18 \\
\hline Poaceae & 7 & 5 & 12 \\
\hline Brassicaceae & 1 & 9 & 10 \\
\hline Fabaceae & 1 & 6 & 7 \\
\hline Polygonaceae & 2 & 3 & 5 \\
\hline Cyperaceae & 4 & 0 & 4 \\
\hline Malvaceae & 1 & 3 & 4 \\
\hline Amaryllidaceae & 2 & 1 & 3 \\
\hline Convolvulaceae & 1 & 2 & 3 \\
\hline Papaveraceae & 0 & 3 & 3 \\
\hline Plantaginaceae & 0 & 3 & 3 \\
\hline Solanaceae & 3 & 0 & 3 \\
\hline
\end{tabular}

tres especies aparecen Polygonaceae, Cyperaceae, Malvaceae, Amaryllidaceae, Convolvulaceae, Papaveraceae, Plantaginaceae y Solanaceae, estas doce familias reúnen un $77 \%$ de la riqueza de especies (véase Tabla 2); entre las demás familias, cuatro contienen dos especies, y 16, solo una. Desde el punto de vista de los géneros, Bromus y Malva contienen tres especies cada uno mientras que los géneros Apium, Brassica, Distichlis, Fumaria, Nolana, Oxalis, Plantago, Polygonum, Rumex, Schoenoplectus, Senecio, Sonchus y Vicia tienen dos especies en los relevamientos.

Origen geográfico. De las 98 especies registradas, 35 son nativas ( $36 \%$ ), 11 de las cuales son endémicas de Chile; las especies alóctonas asilvestradas alcanzaron a 60 , correspondiendo al $61 \%$ de la riqueza del área de estudio; para tres especies clasificada a nivel de género no se pudo determinar el origen geográfico. Entre las familias con mayor número de especies nativas están Poaceae y Cyperaceae, mientras que las familias con mayor riqueza de alóctonas son Asteraceae y Brassicaceae (Tabla 2).

En cuanto al origen de las especies alóctonas asilvestradas, sus distribuciones originales más frecuentes fueron, Europa $(31,7 \%)$, Eurasia (25\%) y el sur de África (8,3\%), estando los demás orígenes representados por menos de un $7 \%$ de las especies (Tabla 3); si se consideran todos los orígenes relacionados con Europa (Europa+Eurasia), a dicha región corresponde el $78 \%$ de ellas (Tabla 3 ).
Tabla 3. Distribución del origen de la flora alóctona.

\begin{tabular}{|c|c|c|}
\hline Distribución original & $\begin{array}{c}\text { Número de } \\
\text { especies }\end{array}$ & $\mathbf{\%}$ \\
\hline Europa & 19 & 31,7 \\
\hline Eurasia & 15 & 25,0 \\
\hline África del Sur & 5 & 8,3 \\
\hline Eurasia y América del Norte & 4 & 6,7 \\
\hline Europa mediterránea y & 4 & 6,7 \\
\hline norte de África & 3 & 5,0 \\
\hline América del Norte & 2 & 3,3 \\
\hline Sur de Europa & 2 & 3,3 \\
\hline Nueva Zelandia & 1 & 1,7 \\
\hline Cosmopolita & 1 & 1,7 \\
\hline Europa y norte de África & 1 & 1,7 \\
\hline Sur de Europa, & 3 & 5,0 \\
\hline norte de África e India & $\mathbf{6 0}$ & $\mathbf{1 0 0}$ \\
\hline Indeterminado & & \\
\hline Total & & \\
\hline
\end{tabular}

Tabla 4. Formas de vida de la playa Grande de Las Cruces, Chile. Entre () se incluye el total de especies, considerando que no se pudo determinar el origen de tres especies.

\begin{tabular}{|c|c|c|c|}
\hline Forma de vida & $\begin{array}{c}\text { Total de } \\
\text { especies }\end{array}$ & $\begin{array}{c}\text { Alóctonas } \\
\text { asilvestradas }\end{array}$ & $\begin{array}{c}\text { Nativas y } \\
\text { Endémicas }\end{array}$ \\
\hline Terófita & $39(41)$ & 32 & 7 \\
\hline Hemicriptófita & $38(39)$ & 20 & 18 \\
\hline Nanofanerófita & 9 & 5 & 4 \\
\hline Geófita & 3 & 1 & 2 \\
\hline Caméfita & 5 & 1 & 4 \\
\hline Fanerófita & 1 & 1 & 0 \\
\hline Sin determinar & 3 & - & - \\
\hline Total & $\mathbf{9 5}(\mathbf{9 8})$ & $\mathbf{6 0}$ & $\mathbf{3 5}$ \\
\hline
\end{tabular}

Formas de vida. La flora del área de estudio está formada por 41 terófitas (42\%), incluyendo hierbas anuales y bienales, 39 hemicriptófitas (40\%), nueve nanofanerófitas $(9 \%)$ entre las que se encuentran arbustos siempreverdes y caducifolios en verano, cinco caméfitas $(5 \%)$ que son principalmente arbustos bajos, tres geófitas (3\%) o hierbas perennes con órganos de reserva subterráneos y apenas un $1 \%$ de fanerófitas (Myoporum laetum). Entre las especies exóticas (alóctonas asilvestradas) predominan en riqueza las terófitas; entre las nativas, las caméfitas y las geófitas (Tabla 4).

\section{Vegetación}

La vegetación se clasificó en cinco unidades (Tabla 5): 


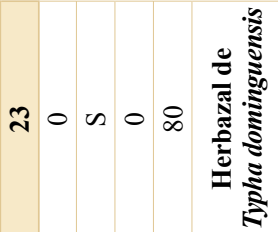

กิน, 0 ฉ

ㄴ. 0 \&

$\infty$ ณ. 0 \&

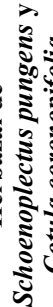

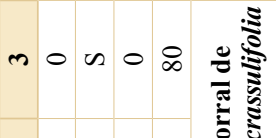

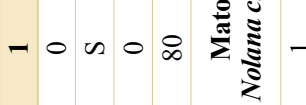

그잉

Iㅇ. 0 i

aㅇ.

nn n

+n u용

느요

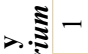

ส으, 0 요

드. 응

느. 108

nn 3 in 8

2 으.으요

Iㅇ. 요 in

$\exists n 3 n$.

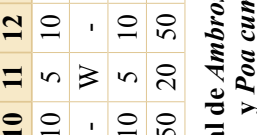

$\infty$ 의 0 우

nㅇ. 10 치

on 10 ?

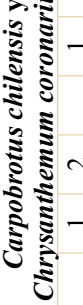

$m \mathrm{n}-\mathrm{N} N \mathrm{~N}-1$
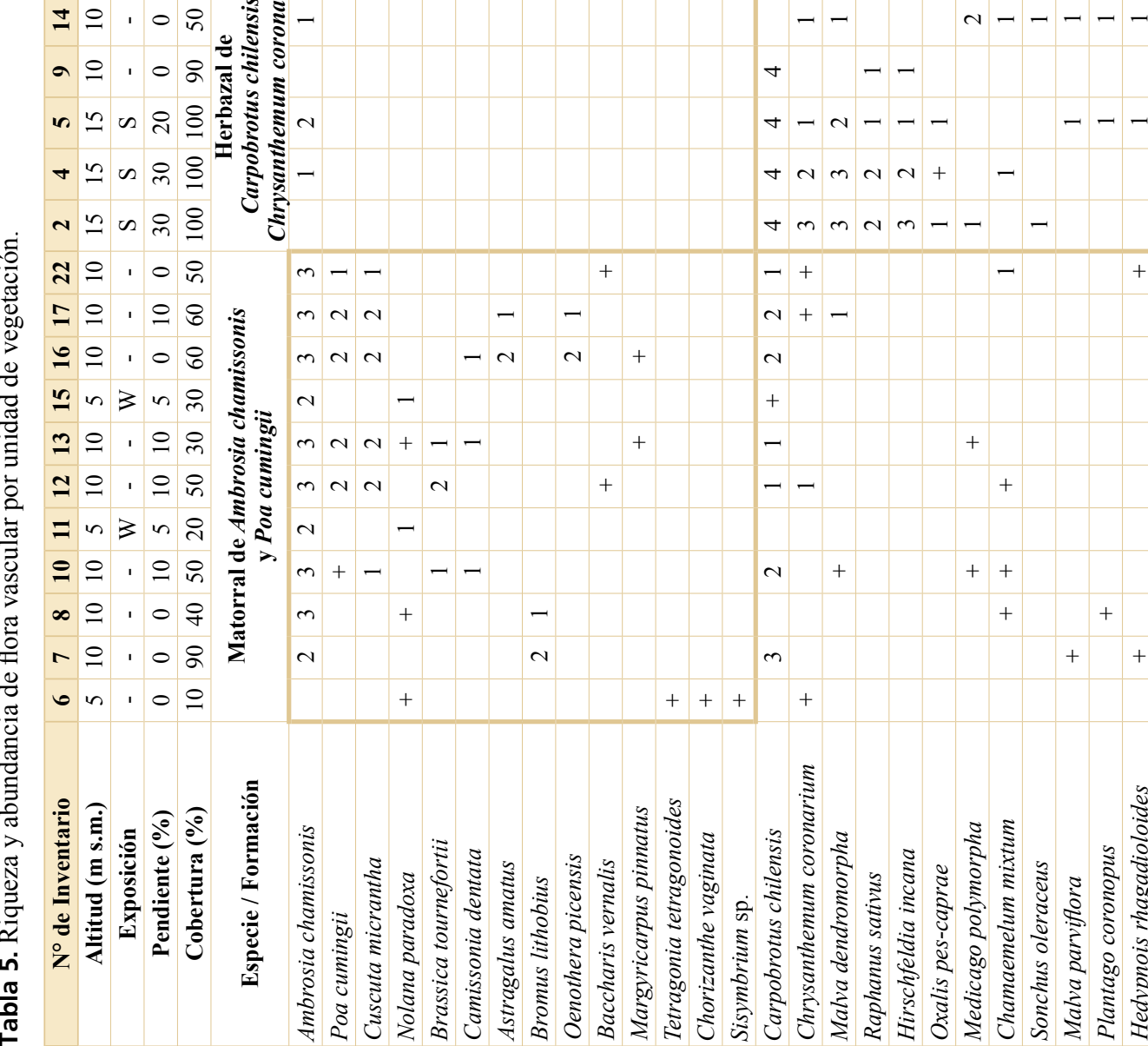

\begin{tabular}{|c|c|c|c|c|c|c|c|c|c|c|c|c|c|}
\hline$n$ & - & - & & & & & & & + & & & & \\
\hline$m$ & $N$ & $N$ & & & & - & & - & & & & & \\
\hline$m$ & $N$ & $N$ & & & - & $N$ & & $N$ & & + & & & \\
\hline$N$ & & & - & & & & & & & & & & \\
\hline$n$ & $N$ & $N$ & + & - & - & & & & & + & & & \\
\hline$m$ & $N$ & $N$ & & $N$ & & & & & + & & & & \\
\hline$N$ & & & - & & & & & & & & & & \\
\hline$m$ & + & - & & - & - & & & & & & & & \\
\hline$m$ & & & + & & & & - & & & & & & \\
\hline$N$ & & & & & & & $v$ & & & & & & \\
\hline & & & + & & & & & & & & & + & + \\
\hline
\end{tabular}




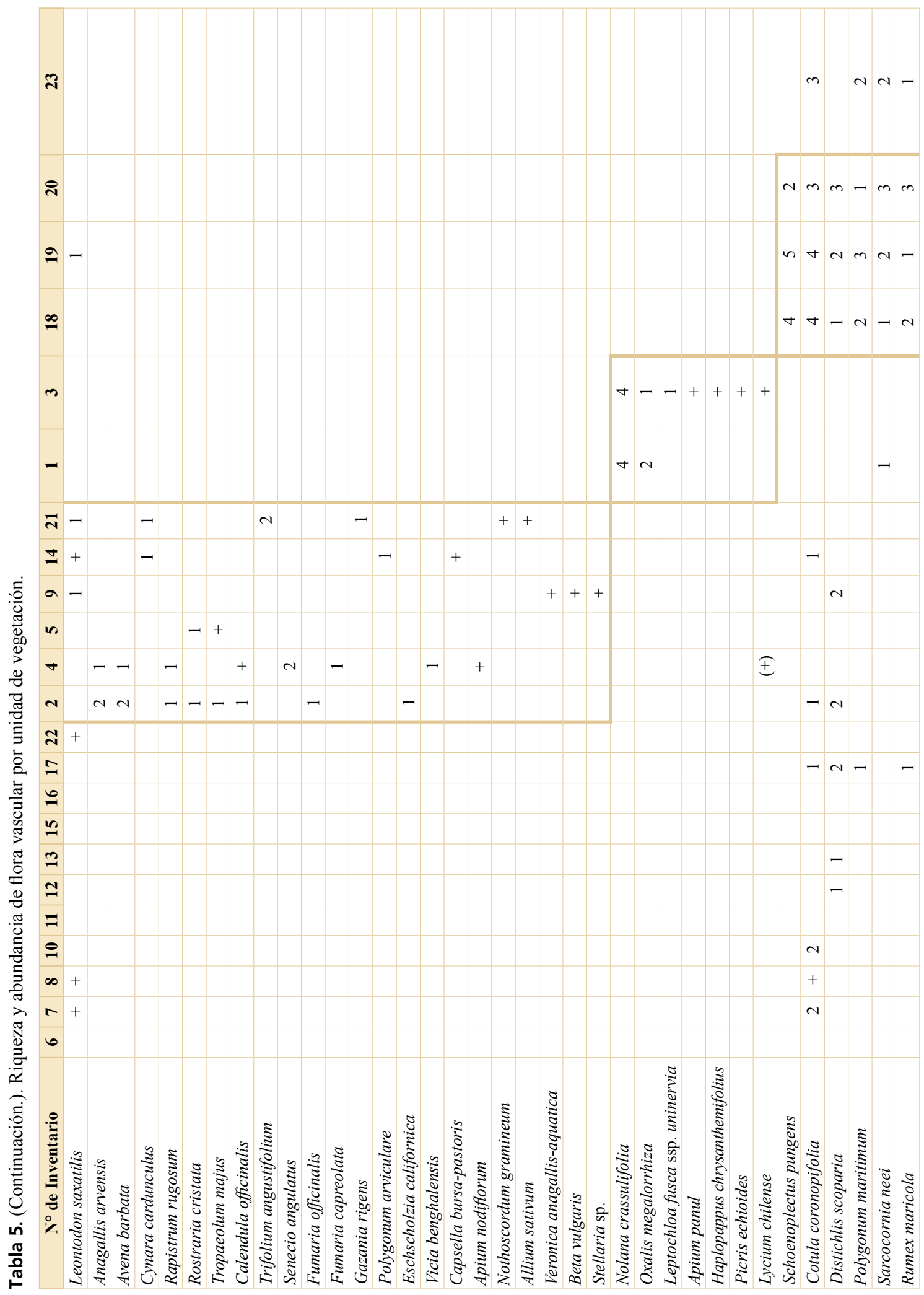




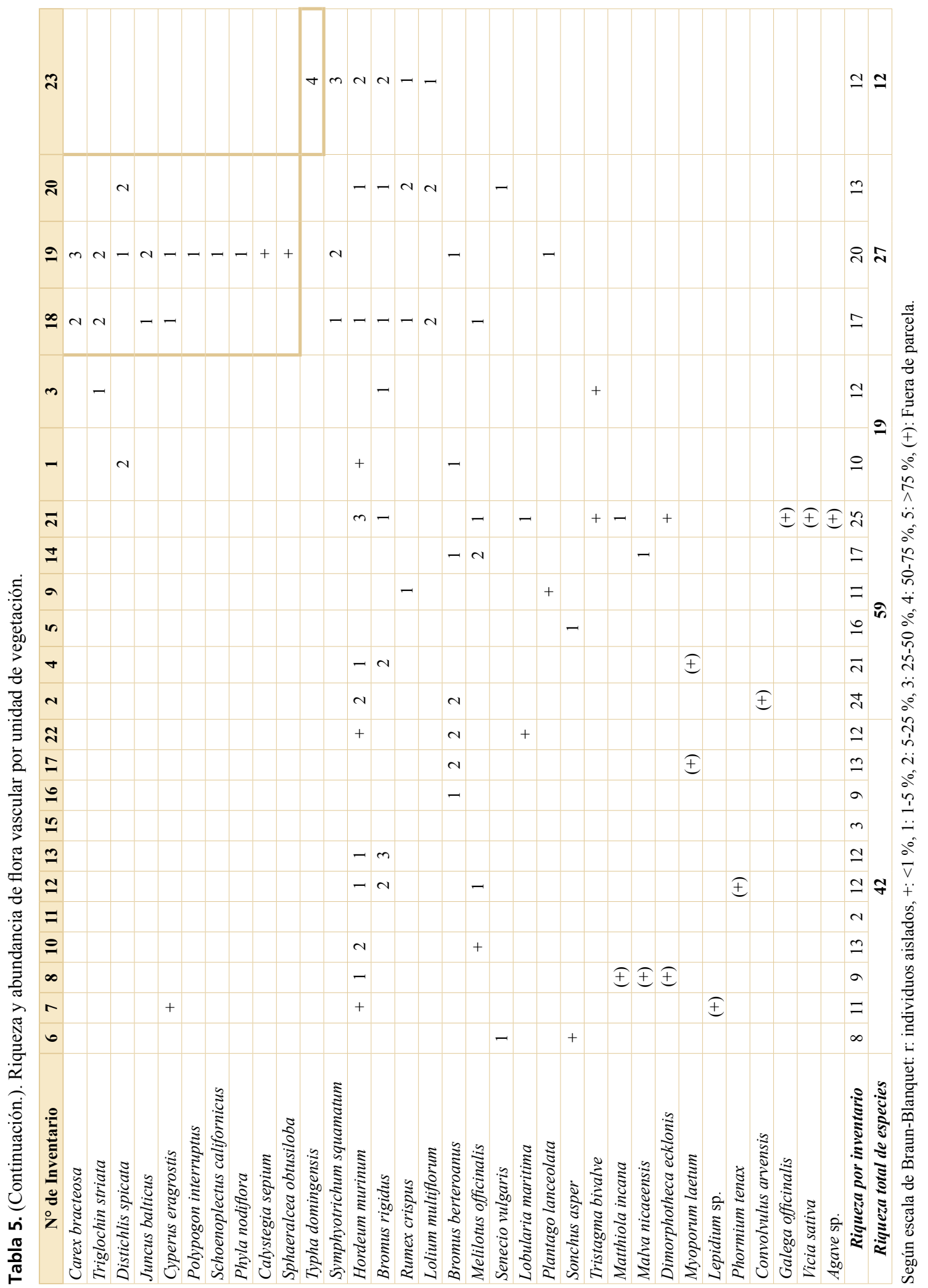


Unidad del matorral de Ambrosia chamissonis y Poa cumingii (Fig. $2 \mathrm{A1}$ y A2). Ocupa los sustratos altamente móviles de la duna holocénica libre, la que incluye tanto las dunas primarias como las secundarias. En la composición de especies figuran, entre otras, las nativas, Carpobrotus chilensis, Poa cumingii, Cuscuta micrantha, Nolana paradoxa, Bromus lithobius, Camissonia dentata, Astragalus amatus, Oenothera picensis, Baccharis vernalis y Margyricarpus pinnatus y las alóctonas asilvestradas, Ambrosia chamissonis y Brassica tournefortii. En relación con la exclusividad de su flora o de la fidelidad de las especies, un $33,3 \%$ de ellas se registraron solo en esta unidad (14 especies). En esta unidad crecían 42 especies de las que 16 (38\%) eran autóctonas y 24 (57\%), alóctonas asilvestradas. La unidad ocupa 6,5 ha, un $76,6 \%$ de la superficie donde crecía algún tipo de vegetación.

\section{Unidad del herbazal de Carpobrotus} chilensis y Chrysanthemum coronarium (Fig. 2B). Comunidad pratense, ruderal, heliófila, localizada en los bordes de las calles que rodean al campo de dunas. Se caracteriza por la presencia y la dominancia de la suculenta nativa Carpobrotus chilensis, acompañada por las especies alóctonas asilvestradas, Chrysanthemum coronarium, Malva dendromorpha, Raphanus sativus, Hirschfeldia incana y Oxalis pes-caprae. Alcanza la mayor riqueza y proporción de exóticas asilvestradas en el área de estudio y en su composición destacan varias alóctonas ornamentales, algunas de ellas en proceso de volverse silvestres tales como Calendula officinalis, Gazania rigens, Lobularia maritima, Myoporum laetum, Senecio angulatus y Tropaeolum majus. En relación con la exclusividad de su flora o de la fidelidad de las especies, un 50,8\% de ellas se registraron solo en esta unidad (30 especies). En la unidad crecían 59 especies, de ellas $6(10 \%)$ eran autóctonas y 51 (86\%), alóctonas asilvestradas. La unidad ocupa 1,3 ha, un 15,6\% de la superficie donde crecía algún tipo de vegetación.

Unidad del matorral de Nolana crassulifolia (Fig. 2C). Es la comunidad de vegetación que crece más cerca del mar, en los roqueríos litorales de Las Cruces. La especie dominante es la solanácea nativa Nolana crasulifolia, un arbusto con hojas suculentas y tolerante a la salinidad, el que, además, es diferencial respecto de las otras unidades; entre sus acompañantes destacan las también nativas, Oxalis megalorriza, Haplopappus chrysanthemifolius, Lycium chilense y Apium panul; salvo O. megalorriza, la mayoría de ellas alcanza una cobertura baja o un número escaso de ejemplares. En relación con la exclusividad de su flora o de la fidelidad de las especies, un 31,6\% de ellas se registró solo en esta unidad (seis especies). La riqueza alcanzó a 19 especies, de las que 13 (68\%) eran autóctonas y 5 (26\%) alóctonas asilvestradas. La unidad ocupa apenas 0,06 ha, menos de un $1 \%$ de la superficie con vegetación. El herbazal de Carpobrotus chilensis y Chrysanthemum coronarium, también tolerante a la salinidad y con el que a menudo viene en contacto, ha reducido sensiblemente su superficie.

Unidad del herbazal de Schoenoplectus pungens y Cotula coronopifolia (Fig. 2D). Es una de las comunidades que ocupa las dunas húmedas. Se encontró en la depresión del curso de agua de la quebrada Los Helechos (desagüe de laguna El Peral, santuario de la naturaleza, localizado a menos de $1 \mathrm{~km}$ aguas arriba) en su desembocadura en el mar, lo que le confiere condiciones de vegetación de marisma. El área ocupada es un pantano con anegamiento temporal, donde son características las higrófilas (helófitas) nativas, Schoenoplectus pungens, S. californicus, Juncus balticus, Carex bracteosa y Cyperus eragrostis, a los que las acompañan nativas como Sarcocornia neei, un indicador de vegetación de marisma, Rumex maricola y Distichlis scoparia; entre las alóctonas crecen, Cotula coronopifolia y Medicago polymorpha. En relación con la exclusividad de su flora o de la fidelidad de las especies, un 29,6\% de ellas se registraron solo en esta unidad (ocho especies). La riqueza alcanzó a 27 especies, de las que 15 (55\%) eran autóctonas y 12 (45\%), alóctonas asilvestradas. La unidad ocupa 0,58 ha, un $6,8 \%$ de la superficie donde crecía algún tipo de vegetación. 

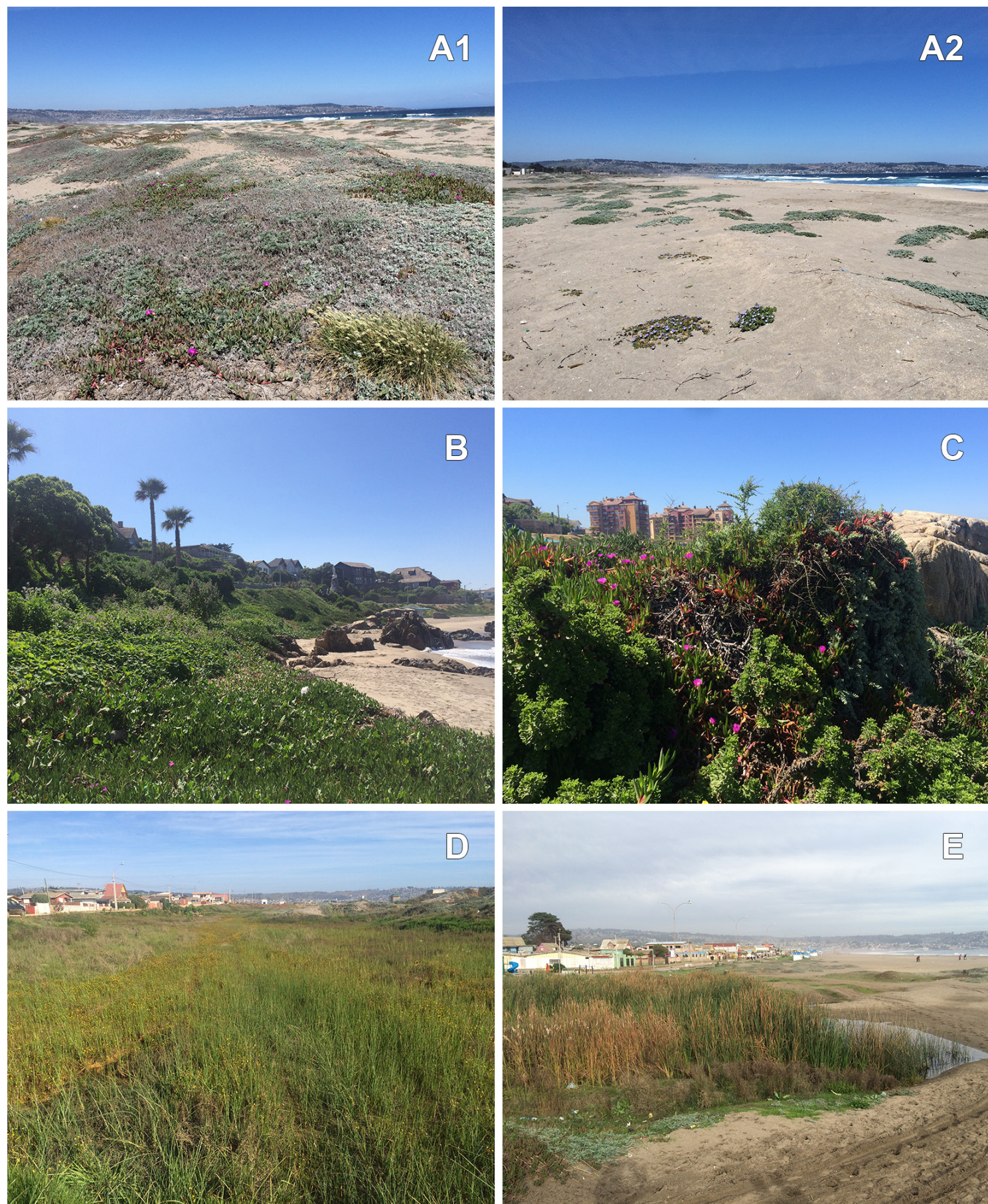

Fig. 2. Fotografías de las unidades de vegetación del área de estudio. A1 y A2, matorral de Ambrosia chamissonis y Poa cumingii. B, herbazal de Carpobrotus chilensis y Chrysanthemum coronarium. C, matorral de Nolana crassulifolia. D, herbazal de Schoenoplectus pungens y Cotula coronopifolia. E, herbazal de Typha dominguensis. Figura en color en la versión en línea http://www.ojs.darwin.edu.ar/index.php/darwiniana/ article/view/959/1238 
Tabla 6. Origen geográfico de las especies en las unidades de vegetación. RT: riqueza total. RN: riqueza de nativas. RE: riqueza de endémicas. RAS: riqueza de alóctonas asilvestradas. SD: sin determinar. S: superficie.

\begin{tabular}{|c|c|c|c|c|c|c|c|}
\hline Unidad de vegetación & RT & $\mathbf{R N}$ & $\mathbf{R E}$ & RAS & SD & S (ha) & $\underset{(\%)}{S}$ \\
\hline Ambrosia chamissonis y Poa cumingii & 42 & 10 & 6 & 24 & 2 & 6,52 & 76,8 \\
\hline Carpobrotus chilensis y Chrysanthemum coronarium & 59 & 5 & 1 & 51 & 2 & 1,33 & 15,4 \\
\hline Nolana crassulifolia & 19 & 9 & 4 & 5 & 1 & 0,06 & 0,7 \\
\hline Schoenoplectus pungens y Cotula coronopifolia & 27 & 12 & 3 & 12 & 0 & 0,58 & 6,9 \\
\hline Typha dominguensis & 12 & 2 & 1 & 9 & 0 & 0,02 & 0,2 \\
\hline
\end{tabular}

\section{Unidad del herbazal de Typha dominguensis} (Fig. 2E). Este tipo de vegetación, llamado localmente "totoral", se encontró solamente en la ribera del estero La Cigüeña y se extiende aguas arriba del estero por ambas riberas. Se diferencia del resto de formaciones por la presencia exclusiva y dominante de Typha dominguensis, hierba perenne rizomatosa, palustre, también son importantes Symphyotrichum squamatum y Cotula coronopifolia junto a otras especies alóctonas asilvestradas, lo que la transforma en la segunda unidad con mayor proporción de especies alóctonas. En relación con la exclusividad de su flora o de la fidelidad de las especies, un 8,3\% de ellas se registró solo en esta unidad (Typha dominguensis). La riqueza de especies alcanzó a 12, de las que 3 (25\%) eran autóctonas y 9 (75\%), alóctonas asilvestradas. La unidad ocupa 0,02 ha, menos de un $1 \%$ de la superficie donde crecía algún tipo de vegetación.

\section{Riqueza de especies en las unidades de vegetación. En la Tabla 6 se muestran los} datos de riqueza total, de las especies endémicas, nativas, alóctonas asilvestradas y la superficie de cada unidad de vegetación. La mayor riqueza de especies fue registrada en el herbazal de Carpobrotus chilensis y Chrysanthemum coronarium, el que con solo un $15 \%$ de la superficie del área de estudio, es la unidad que alberga la mayor riqueza de especies totales (59), seguida de la unidad de Ambrosia chamissonis y Poa cumingii con 42 especies, la que ocupa la mayor superficie $(77 \%)$. Por el contrario, las unidades de Typha dominguensis y Nolana crassulifolia registraron las menores riqueza, con
12 y 19 especies respectivamente, considerando que también corresponden a las unidades representadas por una menor superficie vegetada, la que alcanza 0,2 y $0,7 \%$ en cada caso.

Origen geográfico de las especies en las unidades de vegetación. El análisis de las nativas, incluidas las endémicas de Chile, muestra que la mayor riqueza se encuentra el matorral de Ambrosia chamissonis y Poa cumingii con 16 especies, seguido por el herbazal de Schoenoplectus pungens y Cotula coronopifolia con 15 especies y la unidad de Nolana crassulifolia con 13. En relación con las especies endémicas de Chile, seis fueron registradas en el matorral de Ambrosia chamissonis y Poa cumingii, cuatro en el de Nolana crassulifolia y tres en el de Schoenoplectus pungens y Cotula coronopifolia.

En relación con la riqueza de las especies alóctonas asilvestradas, en el herbazal de Carpobrotus chilensis y Chrysanthemum coronarium, se registran 59 especies, con un $86 \%$, del total, en tanto que en el otro extremo se encuentra el matorral de Nolana crassulifolia con 19 especies y un $26 \%$ del total (Tabla 6).

Formas de vida en las unidades de vegetación. En dos de las cinco unidades de vegetación las terófitas son la forma de vida con mayor número de especies, sin embargo, en el herbazal de Schoenoplectus pungens y Cotula coronopifolia por crecer en sitios más húmedos, predominan las hemicriptófitas, en las otras dos, la riqueza de terófitas y hemicriptófitas es similar (Tabla 7). 
Tabla 7. Comparación de la riqueza por forma de vida en las diferentes unidades de vegetación.

\begin{tabular}{|c|c|c|c|c|c|}
\hline $\begin{array}{c}\text { Forma } \\
\text { de vida }\end{array}$ & $\begin{array}{c}\text { Matorral de } \\
\text { Ambrosia chamissonis } \\
\text { y Poa cumingii }\end{array}$ & $\begin{array}{c}\text { Herbazal de Carpobrotus } \\
\text { chilensis y Chrysanthemum } \\
\text { coronarium }\end{array}$ & $\begin{array}{c}\text { Matorral } \\
\text { de Nolana } \\
\text { crassulifolia }\end{array}$ & $\begin{array}{c}\text { Herbazal de } \\
\text { Schoenoplectus pungens } \\
\text { y Cotula coronopifolia }\end{array}$ & $\begin{array}{c}\text { Herbazal } \\
\text { de Typha } \\
\text { dominguensis }\end{array}$ \\
\hline Terófita & 19 & 29 & 7 & 7 & 5 \\
\hline Hemicriptófita & 16 & 21 & 7 & 17 & 6 \\
\hline Geófita & 0 & 3 & 2 & 0 & 0 \\
\hline Nanofanerófita & 3 & 5 & 2 & 2 & 0 \\
\hline Caméfita & 3 & 1 & 0 & 1 & 1 \\
\hline Fanerófita & 1 & 1 & 0 & 0 \\
\hline
\end{tabular}

\section{DISCUSIÓN}

\section{Riqueza de especies}

Para las dunas del litoral de Chile, entre

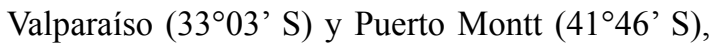
San Martín et al. (1992a) han reportado unas 124 especies nativas, las 35 encontradas en este estudio representan cerca de un tercio de ellas, una riqueza que aparece como alta en comparación con la pequeña superficie levantada. Por otro lado, si se comparan al nivel nacional los resultados respecto de las familias, Asteraceae y Poaceae, las más ricas en especies en este estudio lo son también al nivel del país (Marticorena, 1990). Se destacan localmente por su riqueza las Brassicaceae, esto debido a que dicha familia alberga un número alto de alóctonas asilvestradas: 9 de 10 especies. En términos regionales, la riqueza de las familias coincide, en lo general, con la registrada por Teillier et al. (2010) y García et al. (2019). Lo mismo sucede en términos de la comparación con la flora de las dunas litorales entre Valparaíso y Puerto Montt (San Martín et al., 1992a), donde Poaceae y Asteraceae son las familias que predominan.

\section{Origen geográfico}

El porcentaje de especies alóctonas registradas en el área $(61 \%)$ es considerablemente mayor que el reportado por San Martín et al. (1992a) para las dunas chilenas $(33,3 \%)$ y por Rodríguez et al. (2018) para la flora de Chile (15\%). En gran parte de las dunas litorales del centro de Chile, la flora contiene una alta proporción de especies alóctonas asilvestradas, la que está en relación directa con la intensidad de la alteración antrópica. Comparando la proporción de alóctonas del sitio estudiado con las de otros sitios en la misma región administrativa (Tabla 8) se observa que, por ejemplo, en las desembocaduras del río Aconcagua (Arancibia, 2006) y del estero Reñaca (San Martín et al., 2001), la proporción de especies alóctonas también es alta y alcanza a un $64 \%$ en ambos casos. Al respecto resulta preocupante que, de acuerdo con lo reportado por Jehlík et al. (2019) para los puertos fluviales europeos, la proximidad de la playa Grande de Cartagena con el puerto de San Antonio, el más importante del país (Directemar, 2020), predice un posible incremento de las especies exóticas asilvestradas.

Tabla 8. Comparación del área analizada con otros estudios costeros en Chile central.

\begin{tabular}{|c|c|c|c|c|c|c|c|}
\hline Trabajo & Ubicación & Área & Total & Autóctonas & \% & Exóticas & \% \\
\hline San Martín et al., 1992a & $32-41^{\circ} \mathrm{S}$ & Dunas chilenas & 186 & 124 & 66,7 & 62 & 33,3 \\
\hline Flores \& Araya, 2015 & $32^{\circ} 51^{\prime} \mathrm{S}$ & Dunas de Ritoque & 121 & 95 & 78,5 & 20 & 16,5 \\
\hline Luebert \& Muñoz, 2005 & $32^{\circ} 56^{\prime} \mathrm{S}$ & Dunas de Concón & 159 & 137 & 86,0 & 22 & 14,0 \\
\hline Arancibia, 2006 & $32^{\circ} 54^{\prime} \mathrm{S}$ & $\begin{array}{c}\text { Desembocadura río } \\
\text { Aconcagua }\end{array}$ & 101 & 36 & 36,0 & 65 & 64,0 \\
\hline San Martín et al., 2001 & $33^{\circ} 01^{\prime} \mathrm{S}$ & Estero Reñaca & 73 & 26 & 36,0 & 47 & 64,0 \\
\hline Este estudio & $\mathbf{3 3}^{\circ} \mathbf{3 0} \mathbf{\prime}^{\prime} \mathbf{S}$ & $\begin{array}{c}\text { Tramo norte playa } \\
\text { Grande bahía } \\
\text { Cartagena }\end{array}$ & $\mathbf{9 8}$ & $\mathbf{3 5}$ & $\mathbf{3 5 , 7}$ & $\mathbf{6 0}$ & $\mathbf{6 1 , 0}$ \\
\hline
\end{tabular}


Tabla 9. Características espaciales de las unidades de vegetación.

\begin{tabular}{|c|c|c|c|c|c|}
\hline & $\begin{array}{l}\text { Matorral de } \\
\text { Ambrosia } \\
\text { chamissonis y } \\
\text { Poa cumingii }\end{array}$ & $\begin{array}{c}\text { Herbazal de } \\
\text { Carpobrotus chilensis } \\
\text { y Chrysanthemum } \\
\text { coronarium }\end{array}$ & $\begin{array}{c}\text { Matorral } \\
\text { de Nolana } \\
\text { crassulifolia }\end{array}$ & $\begin{array}{c}\text { Herbazal de } \\
\text { Schoenoplectus } \\
\text { pungens y Cotula } \\
\text { coronopifolia }\end{array}$ & $\begin{array}{c}\text { Herbazal } \\
\text { de Typha } \\
\text { dominguensis }\end{array}$ \\
\hline $\begin{array}{l}\text { Superficie en } \\
\text { hectáreas } \\
\text { (total = 8,51 ha) }\end{array}$ & 6,52 & 1,33 & 0,06 & 0,58 & 0,02 \\
\hline $\begin{array}{l}\text { Superficie de } \\
\text { la unidad como } \\
\text { porcentaje }(\%) \\
\text { del área con } \\
\text { vegetación }\end{array}$ & 76,8 & 15,4 & 0,7 & 6,9 & 0,2 \\
\hline $\begin{array}{l}\text { Número de parches } \\
\quad(\mathrm{n} \text { total }=527)\end{array}$ & 496 & 25 & 2 & 3 & 1 \\
\hline $\begin{array}{l}\text { Promedio en } \\
\text { hectáreas (ha) de } \\
\text { los parches }\end{array}$ & 0,01 & 0,05 & 0,03 & 0,19 & 0,02 \\
\hline
\end{tabular}

Resulta importante señalar también que de las 60 especies alóctonas asilvestradas, al menos once $(18 \%)$ se utilizan en paisajismo. Este aspecto fue estudiado en las dunas costeras del sur de España, donde se encontró que más del $60 \%$ de las especies exóticas silvestres de los ecosistemas costeros psamófilos, correspondían a plantas de jardinería (Asensi et al., 2014). Esta situación también se repetía en el norte de España donde un $35 \%$ de la flora correspondía a especies utilizadas en jardinería (Campos et al., 2004). En ambos casos las familias más importantes eran Asteraceae (25\%) y Poaceae (18\%), las que tienen efectivos mecanismos de dispersión por viento y agua por lo que resultan muy competitivas en ambientes bajo la influencia marina (Campos et al., 2004).

\section{Formas de vida}

En el espectro biológico de Raunkiaer predominan las terófitas (hierbas anuales) con un $41 \%$, seguidas muy cerca por las hemicriptófitas, con un $40 \%$ y luego las nanofanerófitas, con un $11 \%$ de las especies. Este resultado es similar al registrado por San Martín et al. (1992a) para la flora de las dunas de Chile central y sur. Comparativamente, Spanou et al. (2006) reportan que en las dunas mediterráneas de Grecia las terófitas también son las dominantes con un $52 \%$ de las especies. En un ambiente de clima con tendencia mediterránea, el predominio de las terófitas es un resultado esperable cuando se trata de la vegetación de un ambiente árido e inestable como lo son las dunas.
Distribución y grado de fragmentación de las unidades de vegetación. Para las 22,2 ha del área de estudio se reconocieron 527 parches de vegetación de diferente tamaño (Tabla 9) sumando entre todos 8,51 ha. Un $94 \%$ de ellos, con un tamaño promedio de 0,01 ha, se asignó al matorral de Ambrosia chamissonis y Poa cumingii. Este gran número de parches con una superficie tan pequeña da cuenta del alto grado de fragmentación de la vegetación que probablemente se ha producido tanto de manera natural, como debido a la intervención antrópica mediante el pisoteo o el desplazamiento de vehículos por las dunas. De acuerdo con Šilc et al. (2017) el pisoteo es una de las actividades humanas más dañinas para este tipo de ecosistemas, y se ha demostrado que durante acciones de restauración ambiental la eliminación del pisoteo incrementa la riqueza de la flora y amplía la cobertura de la vegetación (Acosta et al., 2013).

Relaciones fitosociológicas. La unidad de vegetación de Ambrosia chamissonis y Poa cummingii se incluye en la asociación Poo-Ambrosietum chamissonis (Kohler, 1970; Eskuche, 1992), donde posiblemente se determinó a Poa cumingii, como $P$. aff. lanuginosa. De acuerdo con Kohler (1970), esta asociación se distribuye desde las dunas del sur de Quintero hasta Constitución (Región del Maule). Ambrosia chamissonis, en particular, es uno de 
los ejemplos más impresionantes de neófitos en Chile, ya que esta planta ha logrado alterar el aspecto de la vegetación de las dunas y playas en más de 1500 km (Kohler \& Weisser, 1966), ya que aparentemente ha logrado desplazar a Carpobrotus chilensis que, como describe Albert (1900), hasta fines del siglo XIX era la principal colonizadora de las dunas costeras. La cobertura de A. chamissonis en California, Estados Unidos, ha sido reportada como mayor en las playas con alto nivel de impacto por lo que, al parecer, ocupa un nicho ambiental en el que otras especies tienen dificultades para sobrevivir (Tobias, 2013). Además, Ambrosia chamissonis es capaz de mantenerse sobre sustratos móviles debido a que puede resistir cierto grado de cubrimiento por la arena (Kohler \& Weisser, 1966).

Desde el punto de vista sintaxonómico la unidad se clasifica como:

Clase: Ambrosietea chamissonis Kohler, 1970

Orden: Ambosietalia chamissonis Kohler, 1970

Alianza: Polygonion sanguinariae Kohler, 1970

Asociación: Poo-Ambrosietum chamissonis Kohler, 1970

El herbazal de Schoenoplectus pungensCotula coronopifolia podría tratarse como una variante de la asociación propuesta por San Martín et al. (1992b) como Scirpo-Cotuletum coronopifolia, los que señalan que tiene un carácter ecotonal entre duna y marisma. A nivel local, la diferencia entre ambas unidades es que la dominante es Schoenoplectus pungens en lugar de Scirpus americanus (=Schoenoplectus americanus), aunque esto puede deberse incluso a algún error en las determinaciones de las especies de este género complejo.

Desde el punto de vista sintaxonómico esta unidad se clasifica en:

Clase: Phragmito-Magnocaricetea Klika in Klika \& Novák, 1941

Orden: Scirpetalia californici San Martín, Medina, Ojeda \& Ramírez, 1993

Alianza: Scirpion californici San Martín, Medina, Ojeda \& Ramírez, 1993

Asociación: Scirpo-Cotuletum coronopifoliae San Martín et al., 1992
El matorral rupícola de Nolana crassulifolia, podría tratarse de una variante empobrecida de lo propuesto por Luebert (2005) como Bahio ambrosiodis-Nolanetum crassulifolia, pero con un menor número de especies y la ausencia local de Bahia ambrosioides, y que continúa manifestándose por los roqueríos costeros hacia el noroeste del área de estudio.

Desde el punto de vista sintaxonómico esta unidad se clasifica como:

Clase: Gutierrezio-Trichocereetea Oberdorfer, 1960

Orden: Saturejo-Puyetalia chilensis Balduzzi, Serey, Tomaselli \& Villaseñor, 1981

Alianza: Carpobroto aequilateri-Baccharidion macraei Luebert, 2005

Asociación: Bahio ambrosioidis-Nolanetum crassulifoliae Luebert, 2005.

Respecto de los herbazales de Carpobrotus chilensis-Chrysanthemum coronarium y de Typha dominguensis, como no han sido formalmente reconocidas, no se incluyen en los esquemas de clasificación. Cabe destacar que el herbazal de $T$. dominguensis forma parte de una unidad mayor que se extiende hacia el este por ambas riberas de la quebrada La Cigüeña, pero que en el área de estudio solo está representada por un parche.

A modo de conclusión, los resultados concuerdan con la idea de Melzi et al. (2020), respecto de la importancia que tiene conocer y manejar los neoecosistemas (sensu Hobbs et al., 2006). En el país, las especies nativas y las alóctonas asilvestradas a lo largo de 500 años de convivencia han ido creando nuevas combinaciones, generando equilibrios dinámicos que varían con los cambios en la intensidad de la actividad humana. Al igual que lo reportado por Morello (2000), en muchos casos se establecen estos ecosistemas mixtos donde las especies exóticas invasoras terminan siendo las dominantes o más frecuentes en desmedro de las nativas. Sin embargo, aun así, resulta relevante conocer la composición y la riqueza de estos neoecosistemas puesto que las comunidades vegetales de los sistemas de dunas de Chile central, a pesar del intenso uso antrópico a que han estado sujetos, aun albergan especies endémicas críticamente amenazadas. 


\section{A. MADRID \& S. TEILLIER. Flora y vegetación de la Playa Grande de Cartagena, Chile}

\section{AGRADECIMIENTOS}

A Federico Luebert por sus comentarios y contribuciones en la mejora del manuscrito, a Diego Urzúa por la elaboración de la figura 1 y a los revisores anónimos por sus comentarios que lo enriquecieron.

\section{BIBLIOGRAFÍA}

Acosta, A.; T. Jucker, I. Prisco \& R. Santoro. 2013. Passive recovery of Mediterranean coastal dunes following limitations to human trampling. In: Martínez L. M.; J. B. Gallego \& P. A. Hesp (eds.). Restoration of coastal dunes. Springer-Verlag, Berlin Heidelberg, pp. 187-198.

Albert, F. 1900. Las dunas, o sea, las arenas volantes, voladeros, arenas muertas, invasión de las arenas, playas y médanos del centro de Chile. Imprenta Cervantes. 148 pp.

Arancibia, J. 2006. Flora vascular de la desembocadura del río Aconcagua. Anales del Museo de Historia Natural de Valparaíso 25: 34-46.

Asensi, A.; B. Diez-Garretas \& J. Perena. 2014. Alien plants of coastal dune habitats in southern Spain. Plant Biosystems 150(3): 477-483. DOI: https://doi.org/10.1080/11263504.2 014.973463

Braun-Blanquet, J. 1979. Fitosociología, bases para el estudio de las comunidades vegetales. Blume Ediciones, Madrid. $820 \mathrm{pp}$.

Brito, B. 2017. Primeros antecedentes para la conservación de la flora vascular de la quebrada La Hoyada, Las Cruces, Región de Valparaíso, Chile. Anales del Museo de Historia Natural de Valparaíso 29: 67-71.

Caldichoury, R. 2000. Análisis de la Vegetación en Cronosecuencias Dunares Campo de Dunas Santo Domingo - El Yali. Investigaciones geográficas 34: 17-28.

Campos, J. A.; M. Herrera, I. Biurrum \& J. Loidi. 2004. The role of alien plants in the natural coastal vegetation in central-northern Spain. Biodiversity and Conservation 13: 2275-2293. DOI: https://doi.org/10.1023/ B:BIOC.0000047902.27442.92

Castillo, S. \& P. Moreno. 1996. Coastal sand dune vegetation: an extreme case of species invasion. Journal of Coastal Conservation 2: 13-22. DOI: https://doi.org/10.1007/ BF02743033

Castro, C. \& R. Hidalgo. 2002. Del pueblo balneario a la gran conurbación: la expansión urbana en el litoral central de la V Región de Valparaíso, Provincia de San Antonio, 1954-2000. Revista Geográfica de Valparaíso 32-33: 91-103.
Calvã, O.; M. Pessoa \& F. Lidon. 2017. Impact of human activities on coastal vegetation - A review. Emirates Journal of Food and Agriculture 25(12): 926-944. DOI: https://doi. org/10.9755/ejfa.v25i12.16730

Clemants, S. \& G. Moore. 2003. Patterns of species richness in eight northeastern United States cities. Urban Habitats 1: 4-16.

Cowling, R.; C. Logie, J. Brady, M. Middleton \& B. Grobler. 2019. Taxonomic, biological and geographical traits of species in a coastal dune flora in the southeastern Cape Floristic Region: regional and global comparisons. PeerJ 7: e7336. DOI: https://doi.org/10.7717/peerj.7336

Dietrich, W. 1977. The South American species of Oenothera Sect. Oenothera (Raimannia, Renneria; Onagraceae). Annals of the Missouri Botanical Garden 64(3): 425-626.

Directemar. 2020. Boletín Estadístico Portuario. Armada de Chile, Dirección General del Territorio Marítimo y de Marina Mercante. $102 \mathrm{pp}$

Donoso, T. G. 1974. Observaciones preliminares sobre la vegetación de las dunas en Llico. ( $34^{\circ} 46^{\prime} \mathrm{S} ; 7^{\circ} 05^{\prime} \mathrm{W}$ ) Provincia de Curicó. Noticiario Mensual del Museo Nacional de Historia Natural, Chile 212 y 213: 11-13.

Eskuche, U. 1992. La vegetación de las dunas marítimas de América Latina. Bosque 13(1): 23-28.

Figueroa, J.; S. Teillier, N. Guerrero, C. Ray, S. Rivano, D. Saavedra \& S. Castro. 2016. Vascular flora in public spaces of Santiago, Chile. Gayana Botanica 73(1): 85-103. DOI: http://doi.org/10.4067/S0717-66432016000100011

Flores, L. \& M. Araya. 2015. Línea Base Flora. pp. 27-57. Informe Final. Diagnóstico de sitios de alto valor para la conservación en la Región de Valparaíso. Portafolio sitio Dunas de Ritoque. Universidad Católica de Valparaíso y Universidad de Playa Ancha.

Flores, M. \& J. Arancibia. 2017. Informe Diagnóstico de Línea Base de Flora y Avifauna en Campo Dunar de la Playa de Santo Domingo, Chile Central. Fundación Cosmos. 41 pp.

Fuentes, N.; A. Marticorena, A. Saldaña, V. Jerez, J. Ortiz, P. Victoriano, R. Moreno, J. Larraín, C. Villaseñor, G. Palfner, P. Sánchez \& A. Pauchard. 2020. Multi-taxa inventory of naturalized species in Chile. NeoBiota 60: 25-41. DOI: https://doi.org/10.3897/neobiota.60.55366

García, R.; A. Pauchard, E. Fuentes, J. Esquivel, P. Sánchez \& A. Jiménez. 2019. Plantas exóticas en la zona de transición mediterráneo-templada de la Cordillera de la Costa: patrones a escala regional y local. En Biodiversidad y Ecología de los Bosques Costeros de Chile (C. Smith \& F.A. Squeo, eds). Editorial Universidad de Los Lagos. 445-470.

Heyligers, P. C. 2008. Flora of the Stockton and Port Hunter sandy foreshores with comments on fifteen notable introduced species. Cunninghamia 10(3): 493-511. 
Hobbs, R. J.; S. Arico, J. Aronson, J. S. Baron, P. Bridgewater, V. A. Cramer, P. R. Epstein, J. J. Ewel, C. A. Klink, A.E. Lugo, D. Norton, D. Ojima, D. M. Richardson, E. W. Sanderson, F. Valladares, M. Vilà, R. Zamora \& M. Zobel. 2006. Novel ecosystems: theoretical and management aspects of the new ecological world order. Global Ecology and Biogeography 15(1): 1-7. DOI: https://doi. org/10.1111/j.1466-822X.2006.00212.x

Iturriaga, L. 2004. Informe técnico humedal de Tunquén, V Región. Octubre. 41 pp.

Jehlík, V.; J. Dostálek \& T. Frantík. 2019. Alien plants in Central European river ports. NeoBiota 45: 93-115. DOI: https://doi.org/10.3897/neobiota.45.33866

Knapp, R. 1984. Considerations on quantitative parameters and qualitative attributes in vegetation analysis and in phytosociological relevés. En: Knapp, R. (ed.) Sampling methods and taxon analysis in vegetation science. Junk Publishers, La Haya. 77-119.

Kohler, A. \& P. Weisser. 1966. Contribución al problema de los neófitos: Ambrosia chamissonis (Less.) Greene en Chile. Separata Boletín de la Universidad de Chile 69-70: 61-68.

Kohler, A. 1970. Geobotanische Untersuchungen an Küstendünen Chiles zwischen 27 und 42 Grad südl. Breite. Notasches Jahrbuch 9 (1/2): 55-200.

Kohler, A. 1971. La vegetation des dunes maritimes du Chili. Colloques Phytosociologiques 1: 17-23.

Luebert, F. 2005. Comunidades vegetales. En: Dunas de Concón: El desafio de los espacios silvestres urbanos (Elórtegui. S. ed.). pp. 22-27. Taller La Era, Viña del Mar.

Luebert, F. \& M. Muñoz. 2005. Contribución al conocimiento de la flora y vegetación de las dunas de Concón. Boletín del Museo de Historia Natural 54: 11-35.

Luebert, F. \& P. Pliscoff. 2017. Sinopsis bioclimática y vegetacional de Chile. Editorial Universitaria. Santiago. $2^{\text {a }}$ edición. 384 pp.

Marticorena, C. 1990. Contribución a la estadística de la flora vascular de Chile. Gayana Botánica 47(3-4): 85-113.

Martínez, M. \& N. Psuty (eds.). 2004. Coastal Dunes: Ecology and Conservation. Ecological Studies. Vol. 171. SpringerVerlag Berlin Heidelberg. 391 pp.

Melzi Fiorenza, R.; H. Sirolli \& P. Picca. 2020. Vegetación de un área polderizada del Río de la Plata en la Ciudad de Buenos Aires: la Reserva Ecológica Ciudad Universitaria - Costanera Norte. Darwiniana, nueva serie 8(2): 460-478. DOI: https://doi.org/10.14522/ darwiniana.2020.82.908

MMA (Ministerio del Medio Ambiente). 2018. Decreto Supremo No 79/2018 del Ministerio del Medio Ambiente.
Morello, J. H.; G. D. Buzai, C. Baxendale, A. Rodríguez, S. D. Matteucci, R. E. Godagnone \& R. R. Casas. 2000. Urbanización y consumo de tierra fértil. Ciencia Hoy 10(55): 50-61.

Mueller-Dombois, D. \& H. Ellenberg. 1974. Aims and methods of vegetation ecology. Wiley \& Sons, New York, USA. 547 pp.

Paskoff, R. 1970. Recherches geomorphologiques dans le Chili semi-áride. Bordeaux, Biscaye Fréres, 18 pp.

Pérez, C. \& C. Villagrán. 1985. Distribución de abundancias de especies en bosques relictos de la zona mediterránea de Chile. Revista Chilena de Historia Natural 58: 157-170.

Pyšek, P. 1998. Alien and native species in Central European urban floras: a quantitative comparison. Journal of Biogeography 25: 155-163. DOI: https://doi.org/10.1046/ j.1365-2699.1998.251177.x

Ramírez, C.; J. San Martín, C. San Martín \& D. Contreras. 1987. Estudio florístico y vegetacional de la laguna El Peral, Quinta Región de Chile. Revista Geográfica de Valparaiso 18: 105-120.

Ramírez, C.; C. San Martín \& J. San Martín. 1992. Vegetación y dinámica vegetacional en las dunas litorales chilenas. Bosque 13(1): 41-48. DOI: https://doi.org/10.4206/ bosque.1992.v13n1-05

Ramírez, C. \& M. Álvarez. 2012. Flora y vegetación hidrófila de los humedales costeros de Chile. En: J.M. Fariña y A. Camaño (eds.). Humedales costeros de Chile: Aportes cientificos a su gestión sustentable. Ediciones Universidad Católica de Chile, Santiago. pp. 101-145.

Raunkiaer, C. 1934. The Life Forms of Plants and Statistical Plant Geography. Oxford University Press, London. 631 pp.

Rodríguez, R.; C. Marticorena, D. Alarcón, C. Baeza, L. Cavieres, V. L. Finot, N. Fuentes, A. Kiessling, M. Mihoc, A. Pauchard, E. Ruiz, P. Sánchez \& A. Marticorena. 2018. Catálogo de las plantas vasculares de Chile. Gayana Botánica 75(1): 1-430. DOI: http://doi.org/10.4067/ S0717-66432018000100001

San Martín, J.; C. Ramírez \& C. San Martín. 1992a. La flora de las dunas chilenas y sus adaptaciones morfológicas. Bosque 13(1): 29-39. DOI: https://doi.org/10.4206/ bosque.1992.v13n1-04

San Martín, C.; D. Contreras, J. San Martín \& C. Ramírez. 1992b. Vegetación de las marismas del centro-sur de Chile. Revista Chilena de Historia Natural 65(3): 327-342.

San Martín, C.; C. Ramírez, J. San Martín \& R. Villaseñor. 2001. Flora y vegetación del estero Reñaca (V Región, Chile). Gayana Botánica 58(1): 327-342. DOI: http://doi. org/10.4067/S0717-66432001000100004 


\section{A. MADRID \& S. TEILLIER. Flora y vegetación de la Playa Grande de Cartagena, Chile}

Šilc, U.; D. Caković, F. Küzmič \& D. Stešević. 2017. Trampling impact on vegetation of embryonic and stabilised sand dunes in Montenegro. Journal of Coastal Conservation 21: 15-21. DOI: https://doi.org/10.1007/s11852-016-0468-2

Spanou, S.; G. Verroios, G. Dimitrellos, A. Tiniakou \& T. Georgiadis. 2006. Notes on flora and vegetation of the sand dunes of western Greece. Willdenowia 36: 235-246. DOI: https://doi.org/10.3372/wi.36.36119

Teillier, S.; J. Figueroa \& S. Castro. 2010. Especies exóticas de la vertiente occidental de la cordillera de la Costa, Provincia de Valparaíso, Chile central. Gayana Botánica 67(1): 27-43. DOI: http://doi.org/10.4067/S0717-66432010000100004

Teillier, S. \& F. Zuloaga. 2016. Restauración de la especie Astragalus trifoliatus (Fabaceae). Darwiniana, nueva serie 4: 88-90. DOI: https://doi.org/10.14522/ darwiniana.2016.41.709

Teillier, S.; R. Villaseñor, A. Marticorena, P. Novoa \& H. Niemeyer. 2018. Flora del litoral de la Región de Valparaíso. 680 pp.
Thiers, B. 2021 [permanentemente actualizado] Index Herbariorum: a global directory of public herbaria and associated staff. New York Botanical Garden's Virtual Herbarium, http://sweetgum.nybg.org/ih

Tobias, M. 2013. Effect of trampling on Ambrosia chamissonis and Cakile maritima cover on California beaches. Madroño 60(1): 4-10. DOI: https://doi.org/10.3120/0024-9637-60.1.4

Ugalde, D. 2018. Efecto de la expansión urbana sobre el estado de conservación de las dunas costeras de la bahía de Cartagena, Región de Valparaíso. BeGEO 6: 37-50.

Urrutia, N. 2016. Biodiversidad florística y vegetacional del humedal costero Putú, Región del Maule, Chile. Universidad Austral de Chile. Tesis de Ingeniería en Conservación de Recursos Naturales. Valdivia. 96 pp.

Zhao, J.; Z. Ouyang, H. Zheng, W. Zhou, X. Wang, W. Xu \& Y. Ni. 2010. Plant species composition in green spaces within the built-up areas of Beijing, China. Plant Ecology 209: 189-204. DOI: https://doi.org/10.1007/s11258-009-9675-3 


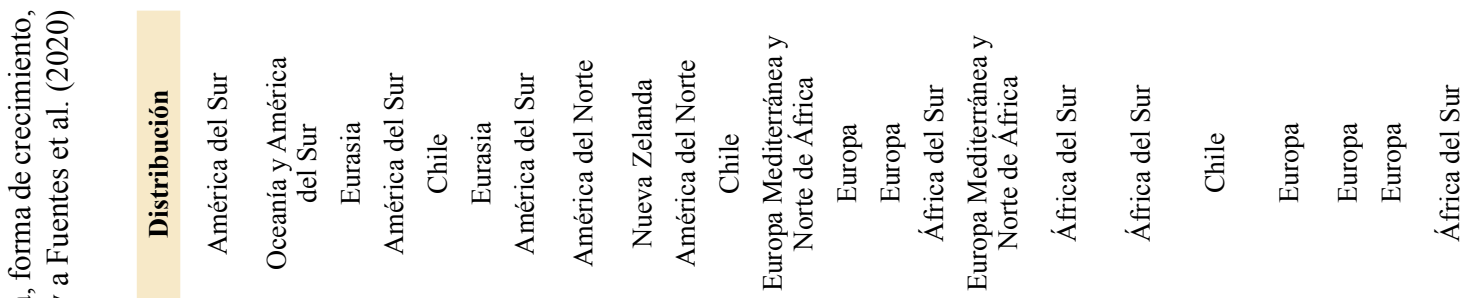

. 7

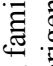

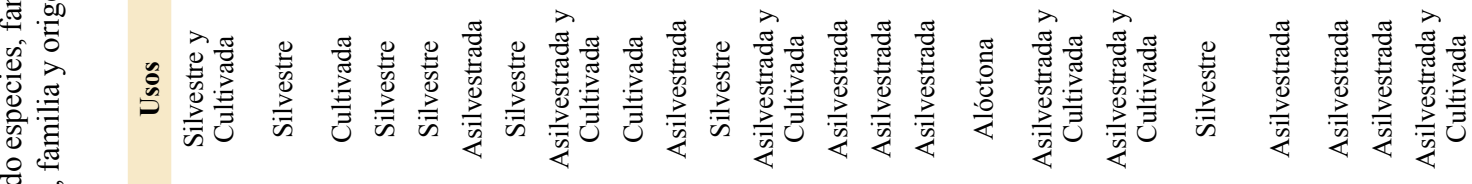

일

产高

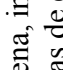

焉言

의

势

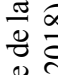

裏

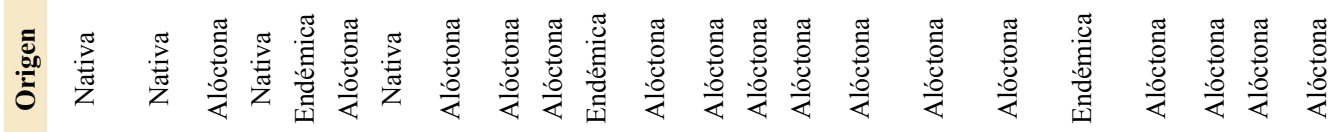

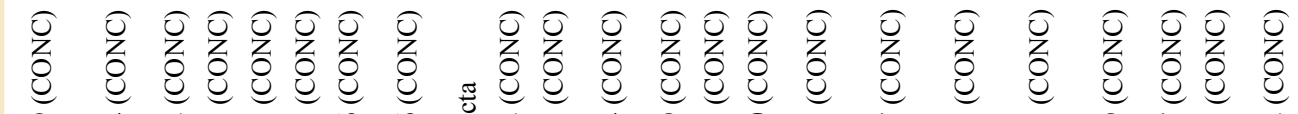

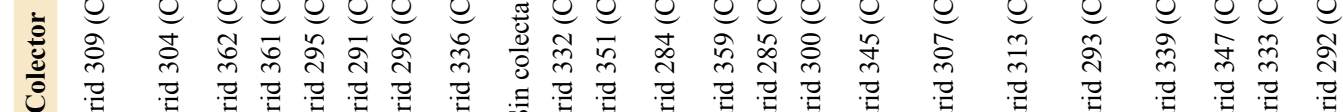

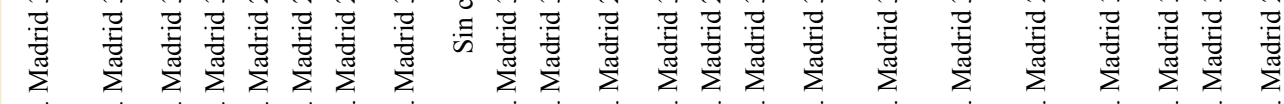

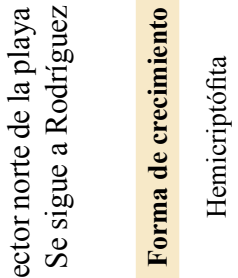

跑

音

商.

営衰

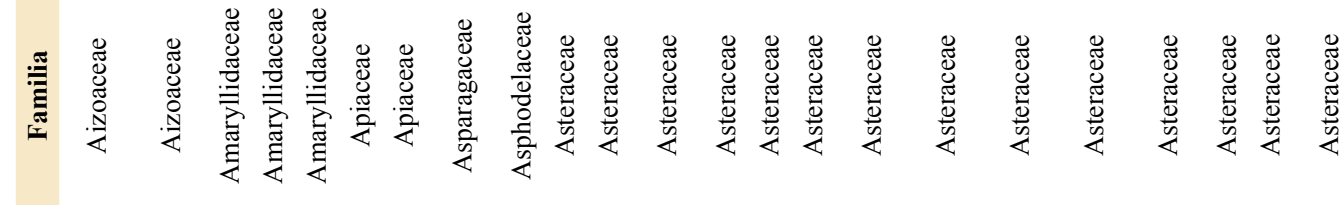

$\frac{9}{9}$

告

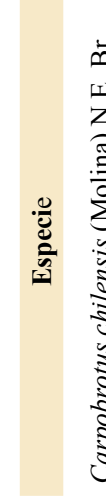

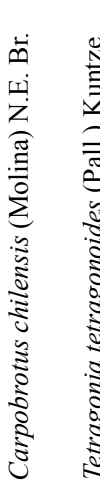

总言

政

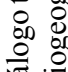

毞

$\dot{\Phi} \bar{b}$

政

栆娄

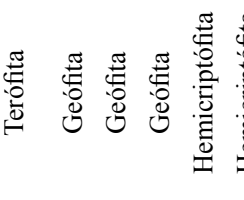

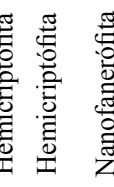

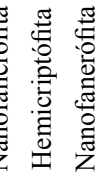

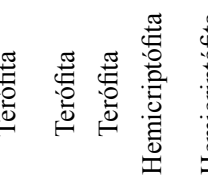

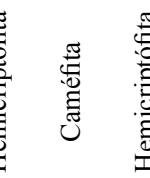

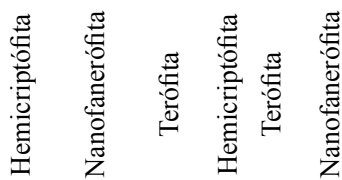

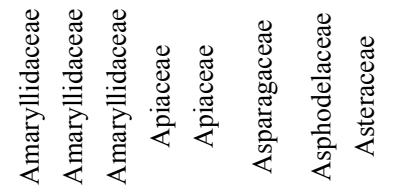

蒙号

๖ 总驾

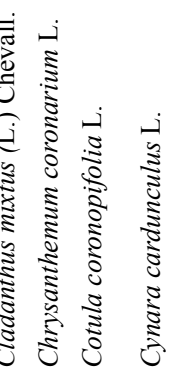

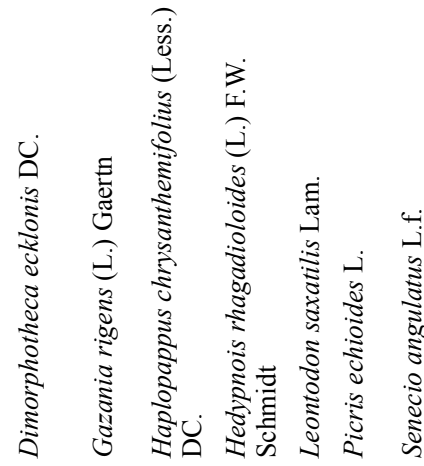


A. MADRID \& S. TEILLIER. Flora y vegetación de la Playa Grande de Cartagena, Chile

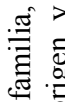

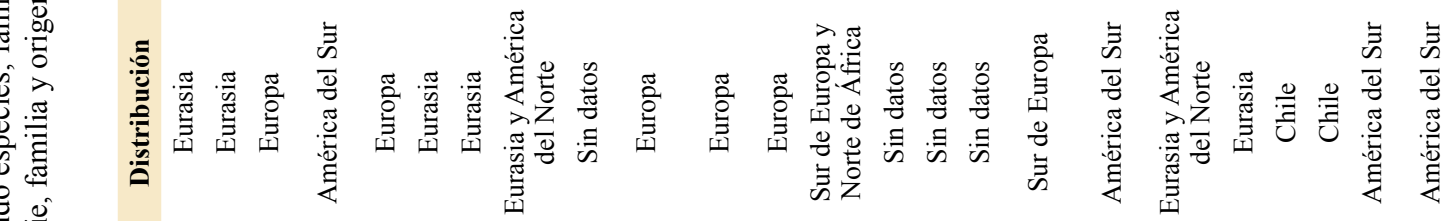

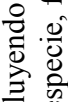

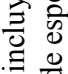

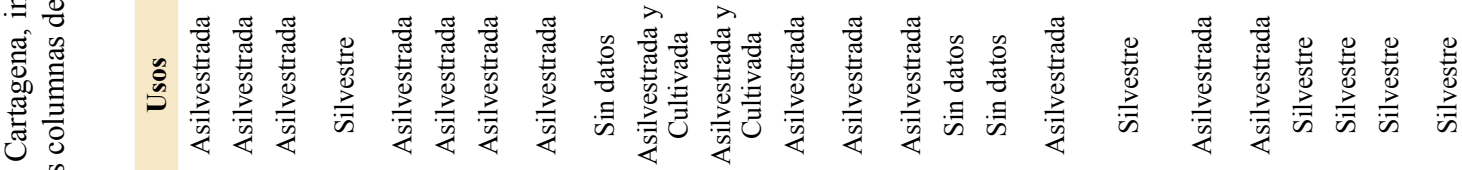

용

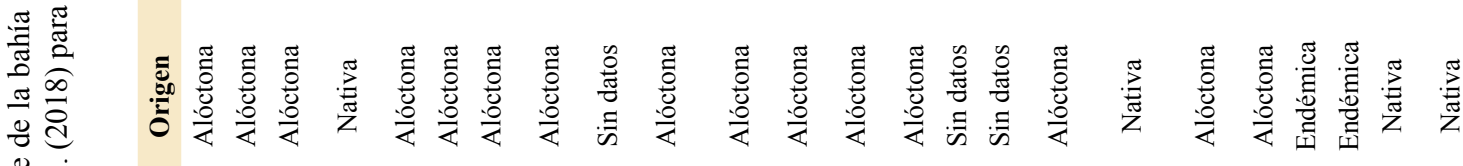

름

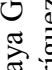

플

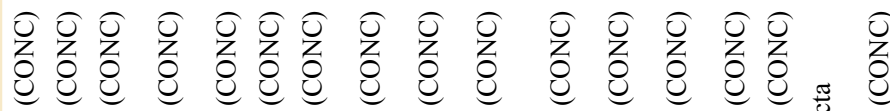

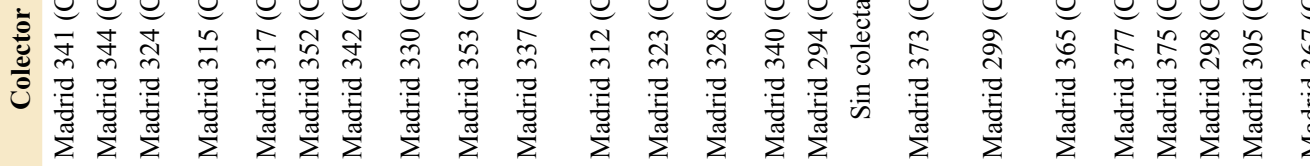

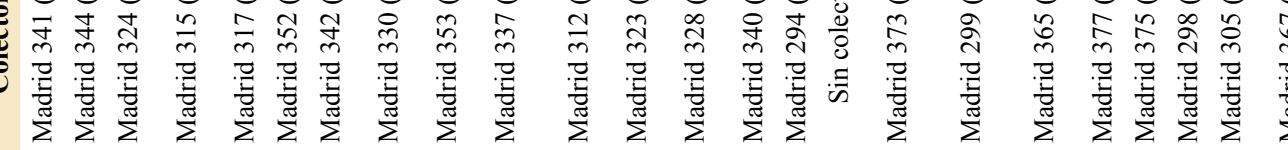

\%

음.

욤․

웜

흘.

产

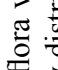

政

.

흥

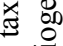

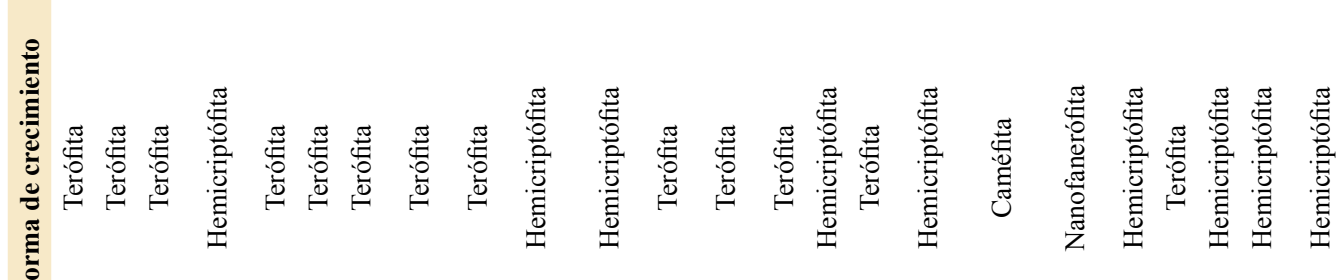

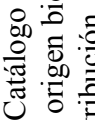

薮

음

音

耐

或

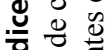

要誉鄫
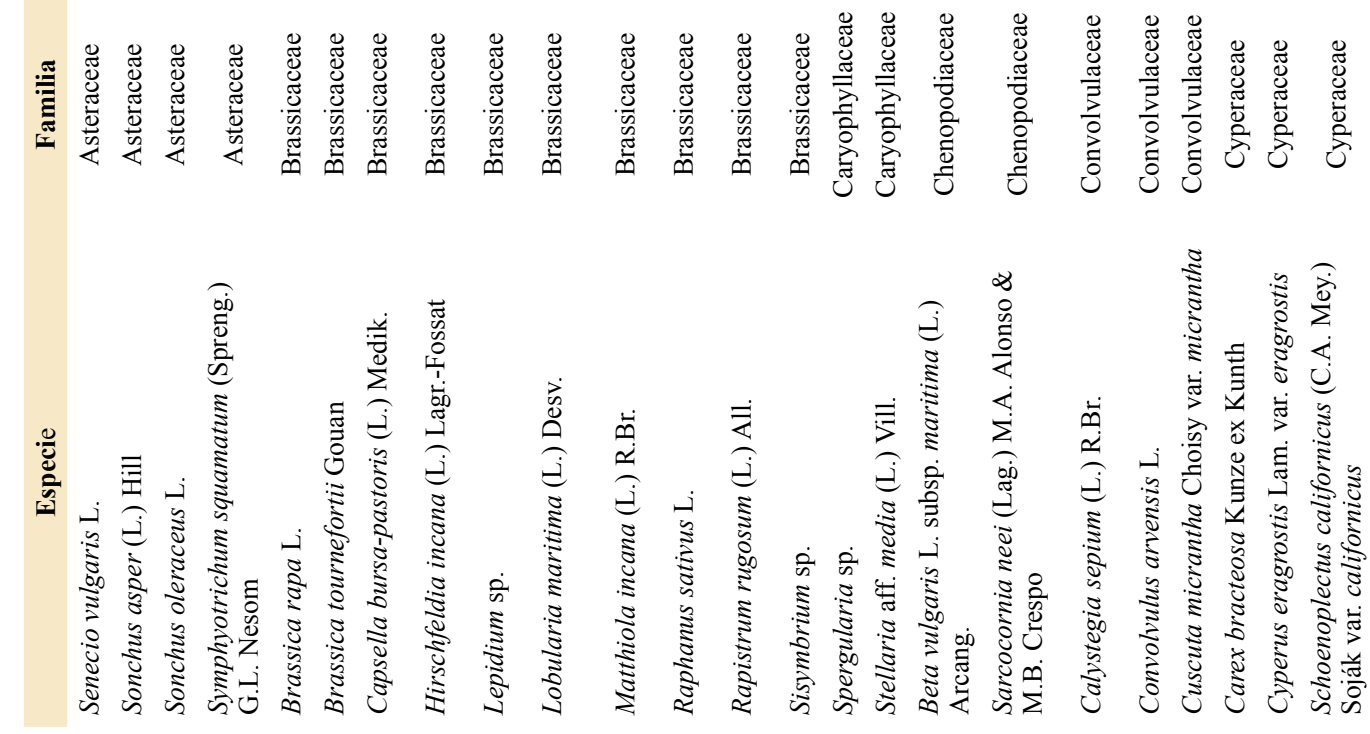
害

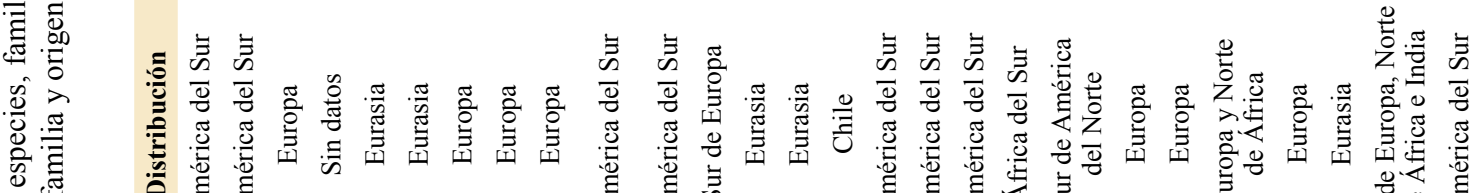

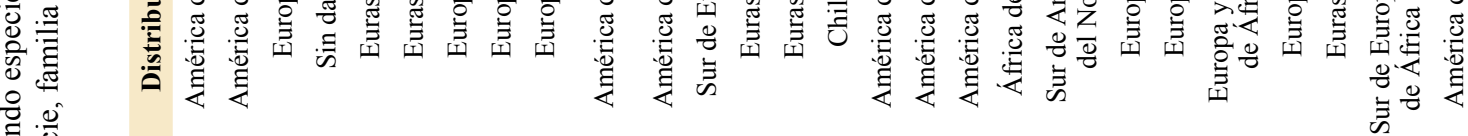

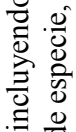

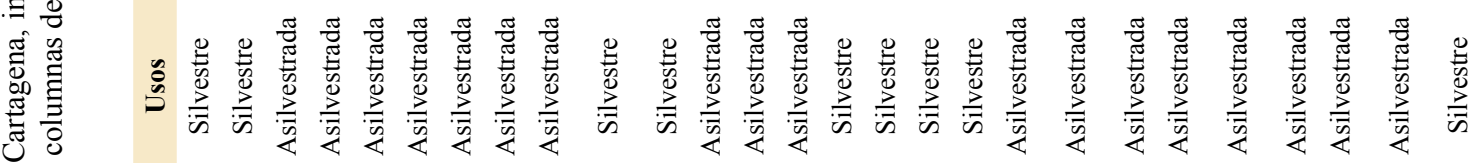

\%

雍

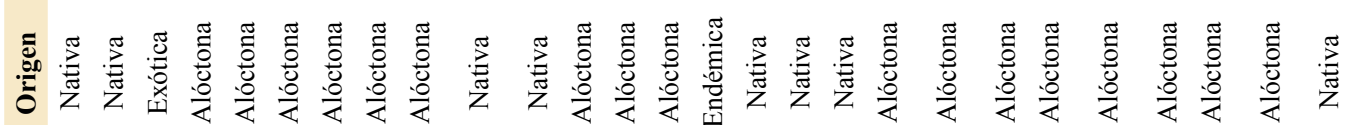

䓪

๑ั

要

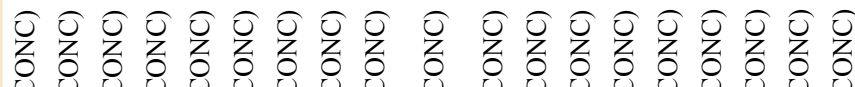

\section{- e e e e e e e}

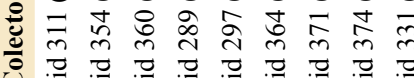

$\frac{0}{2}$

$\frac{\pi}{8}$

:

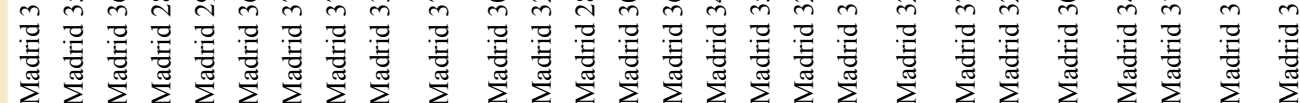

t)

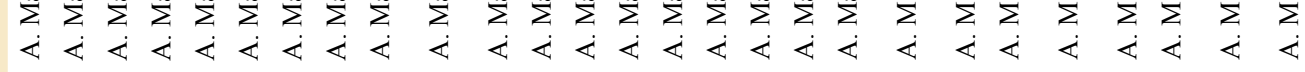

窇

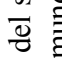

䒕 :
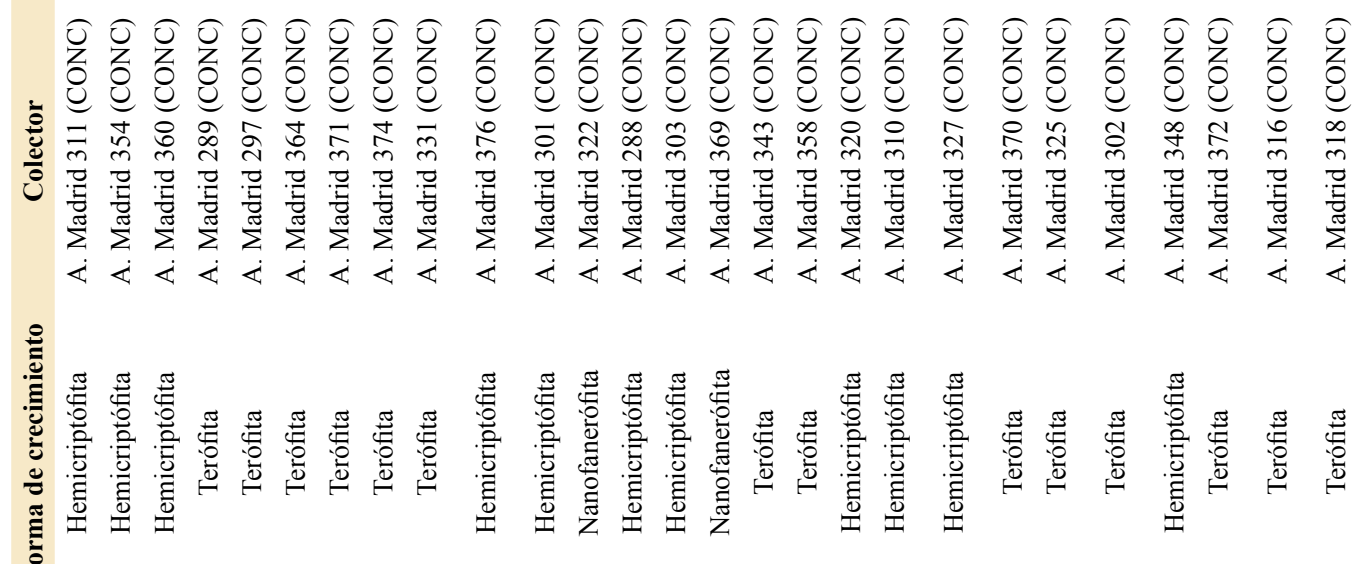

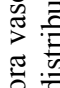

䨔:

픙

.

ㅎํㅇํㅇ 氖

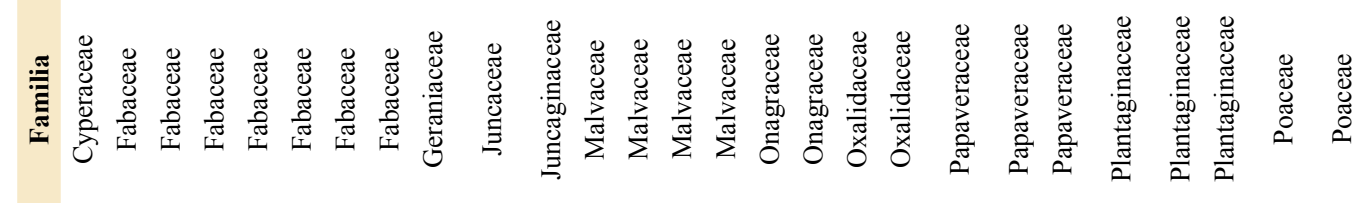

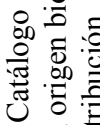

它

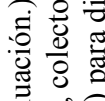

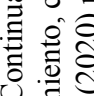

?

๘

쁭

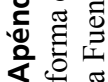

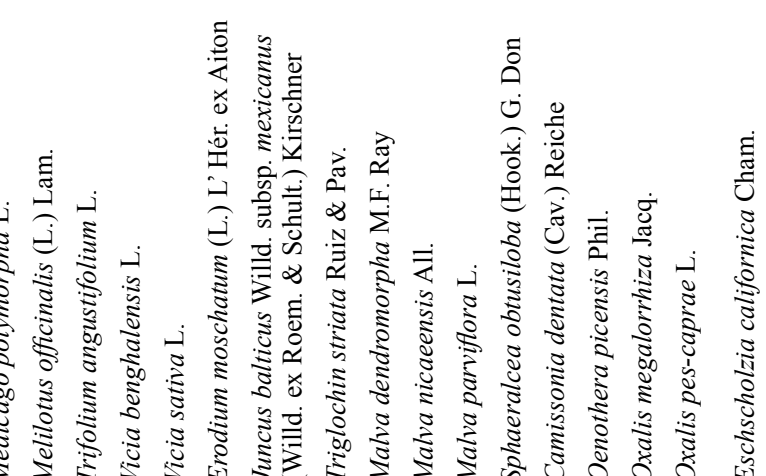

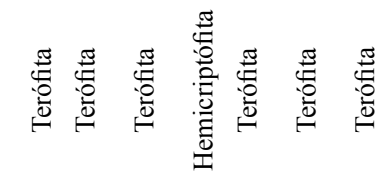<smiles>CCCCCCC</smiles>

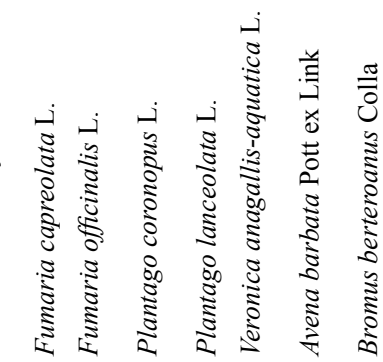


量

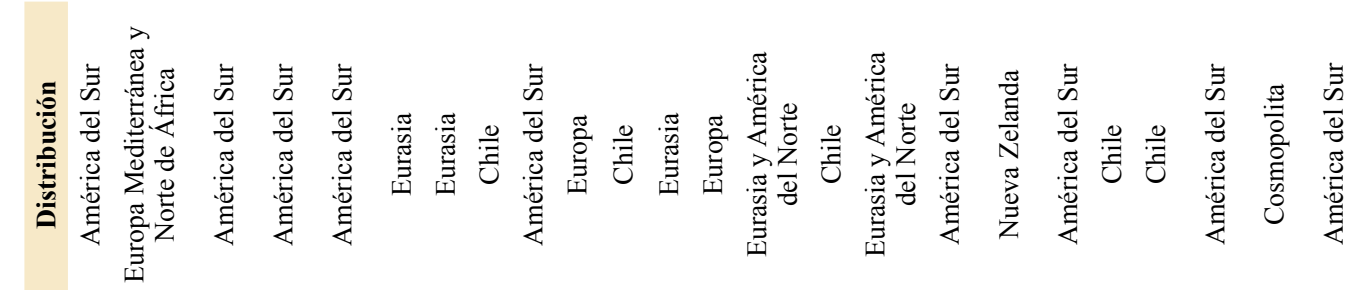

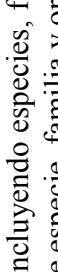

$\exists$

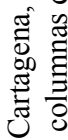

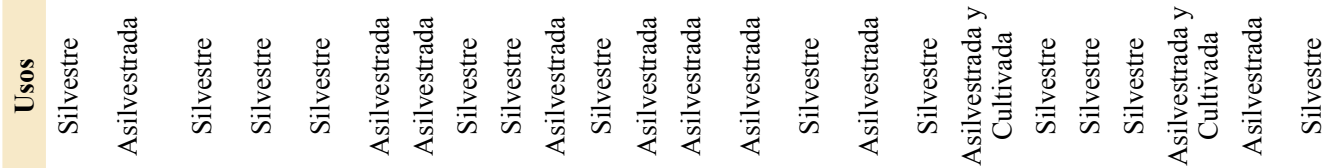

용

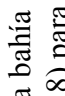

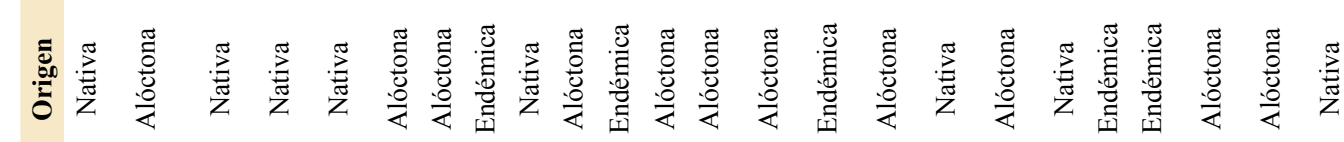

递范

造

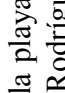

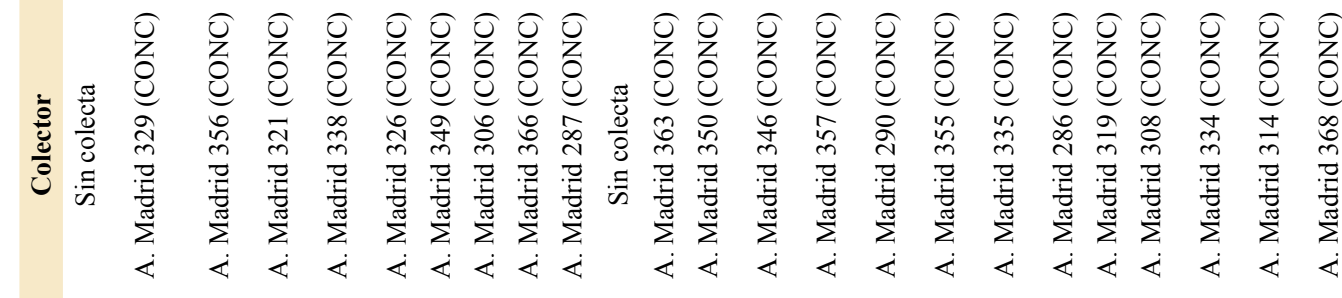

$\frac{\pi}{0} \stackrel{0}{0}$

年

总.

बृ

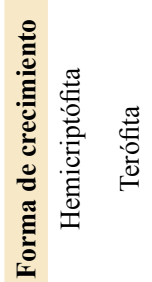

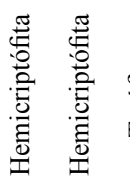

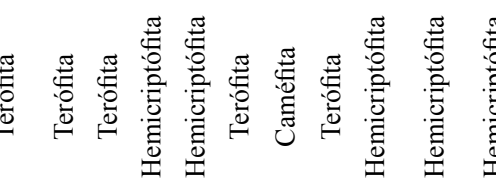

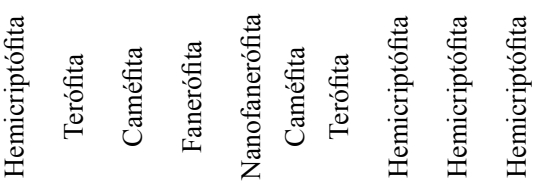

葋步

항

.

II

IIInIIIIIIIIII!I!

的

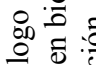

焉.

碂:

:

爱总

过

过苍苋

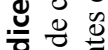

要要总
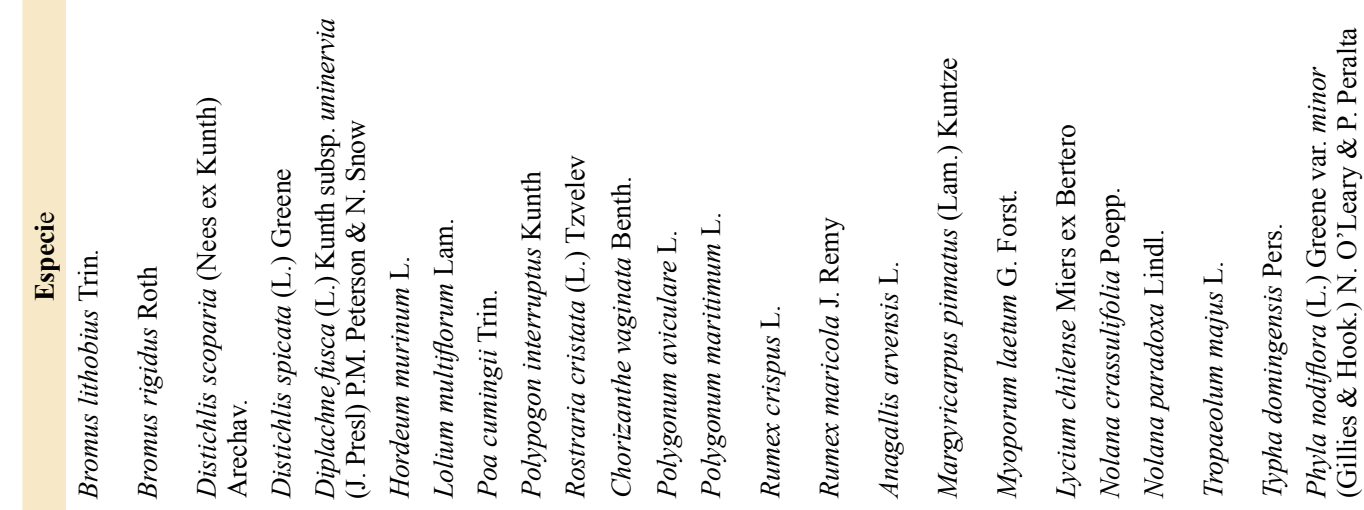